\title{
Strictly proper kernel scores and characteristic kernels on compact spaces*
}

\author{
Ingo Steinwart Johanna F. Ziegel
}

November 21, 2019

\begin{abstract}
Strictly proper kernel scores are well-known tool in probabilistic forecasting, while characteristic kernels have been extensively investigated in machine learning. We first show that both notions coincide, so that insights from one part of the literature can be used in the other. We then show that the metric induced by a characteristic kernel cannot reliably distinguish between distributions that are far apart in the total variation norm as soon as the underlying space of measures is infinite dimensional. We further describe characteristic kernels in terms of eigenvalues and eigenfunctions and apply this characterization to the case of continuous kernels on (locally) compact spaces. In the compact case, we further show that characteristic kernels exist if and only if the space is metrizable. As special cases of our general theory we investigate translation-invariant kernels on compact Abelian groups and isotropic kernels on spheres. The latter are of particular interest for forecast evaluation of probabilistic predictions on spherical domains as frequently encountered in meteorology and climatology.
\end{abstract}

\section{Introduction}

Probabilistic forecasts of uncertain future events are issued in a wealth of applications, see Gneiting and Katzfuss (2014) and the references therein. To assess the quality and to compare such forecasts, proper scoring rules are a well-established tool, see Gneiting and Raftery (2007), and in applications, it is usually even desirable to work with strictly proper scoring rules. A broad class of proper scoring rules are so-called kernel scores, which are constructed using a positive definite kernel. Unfortunately, however, no general conditions are available to decide whether a given kernel induces a strictly proper kernel score. As detailed below in Theorem 1.1, strict propriety of a kernel score is intimately connected to the kernel being characteristic, a notion that has been studied in the machine learning literature for more than a decade, see e.g. Gretton et al. (2007); Fukumizu et al. (2009); Sriperumbudur et al. (2010a, 2011) as well as the recent survey of Muandet et al. (2017) and the references therein. In this paper, we study characteristic kernels on compact spaces extending results of Micchelli et al. (2006) and Sriperumbudur et al. (2010a, 2011). As a consequence, we can characterize strictly proper kernel scores on compact Abelian groups and the practically highly relevant example of spheres.

\footnotetext{
${ }^{*}$ Keywords: Characteristic kernels, Hilbert space embeddings, isotropic positive definite functions on spheres, kernel scores, machine learning, probabilistic forecasts, strictly proper scoring rules, translation-invariant kernels on compact Abelian groups

2010 Mathematics Subject Classification. 33C55, 42A82, 46E22, 47B32, 47B34, 47G10, 62C99, $68 \mathrm{~T} 05$

Declarations of interest: none
} 
To describe our results, let us formally introduce some of the notions mentioned above. To this end, let $(X, \mathcal{A})$ be a measurable space and let $\mathcal{M}_{1}(X)$ denote the class of all probability measures on $X$. For $\mathcal{P} \subseteq \mathcal{M}_{1}(X)$, a scoring rule is a function $S: \mathcal{P} \times X \rightarrow[-\infty, \infty]$ such that the integral $\int S(P, x) \mathrm{d} Q(x)$ exists for all $P, Q \in \mathcal{P}$. The scoring rule is called proper if

$$
\int S(P, x) \mathrm{d} P(x) \leq \int S(Q, x) \mathrm{d} P(x), \quad \text { for all } P, Q \in \mathcal{P},
$$

and it is called strictly proper if equality in (1.1) implies $P=Q$. If $\mathcal{P}$ consists of absolutely continuous probability measures with respect to some $\sigma$-finite measure $\mu$ on $X$, then the logarithmic score $S(P, x):=-\log p(x)$, where $p$ is the density of $P$, is a widely used example of a strictly proper scoring rule for density forecasts. Another well-known example is the Brier score for distributions on $X=\{1, \ldots, m\}$ that is defined as $S(P, i):=\sum_{j=1}^{m} p_{j}^{2}+1-2 p_{i}$, where $p_{i}=P(\{i\})$, $i=1, \ldots, m$. Finally, for $X=\mathbb{R}$, the continuous ranked probability score (CRPS) is given by

$$
S(P, x):=\int_{\mathbb{R}}|y-x| \mathrm{d} P(y)-\frac{1}{2} \int_{\mathbb{R}} \int_{\mathbb{R}}\left|y-y^{\prime}\right| \mathrm{d} P(y) \mathrm{d} P\left(y^{\prime}\right) .
$$

It is strictly proper with respect to the class of all probability measures with finite first moment, see e.g. Gneiting and Raftery (2007, Section 4.2), and consequently it can be used to evaluate predictions of density forecasts as well as probabilistic forecasts of categorical variables. Various other examples can be found in Gneiting and Raftery (2007).

One general class of proper scoring rules are kernel scores. To this end, let $k: X \times X \rightarrow \mathbb{R}$ be a symmetric function. We call $k$ a kernel, if it is positive definite, that is, if

$$
\sum_{i, j=1}^{n} a_{i} a_{j} k\left(x_{i}, x_{j}\right) \geq 0
$$

for all natural numbers $n$, all $a_{1}, \ldots, a_{n} \in \mathbb{R}$, and all $x_{1}, \ldots, x_{n} \in X$. It is strictly positive definite if equality in (1.2) implies $a_{1}=\cdots=a_{n}=0$ whenever the points $x_{1}, \ldots, x_{n}$ are mutually distinct. Let us assume that $k$ is measurable and define

$$
\mathcal{M}_{1}^{k}(X):=\left\{P \in \mathcal{M}_{1}(X) \mid \int_{X} \sqrt{k(x, x)} \mathrm{d} P(x)<\infty\right\} .
$$

For a bounded kernel $k$, we have that $\mathcal{M}_{1}^{k}(X)=\mathcal{M}_{1}(X)$, and the Cauchy-Schwarz inequality $k(x, y) \leq \sqrt{k(x, x)} \sqrt{k(y, y)}$ for kernels shows that, for all $P, Q \in \mathcal{M}_{1}^{k}(X)$, the kernel $k$ is integrable with respect to the product measure $P \otimes Q$.

Definition 1.1. The kernel score $S_{k}$ associated with a measurable kernel $k$ on $X$ is the scoring rule $S_{k}: \mathcal{M}_{1}^{k}(X) \times X \rightarrow \mathbb{R}$ defined by

$$
S_{k}(P, x):=-\int k(\omega, x) \mathrm{d} P(\omega)+\frac{1}{2} \iint k\left(\omega, \omega^{\prime}\right) \mathrm{d} P(\omega) \mathrm{d} P\left(\omega^{\prime}\right) .
$$

Kernel scores are a broad generalization of the CRPS, and in fact, also the Brier score can be rewritten as a kernel score, see Gneiting and Raftery (2007, Section 5.1). However, the logarithmic score does not belong to this class. If $X$ is a Hausdorff space and $k$ is continuous, then Gneiting and Raftery (2007, Theorem 4) show that $S_{k}$ is proper with respect to all Radon probability measures on $X$. Their result is based on Berg et al. (1984, Theorem 2.1, p. 235), where it is fundamental that the kernel is continuous. In this respect we remark that the definition of a 
kernel score of Gneiting and Raftery (2007) is more general than ours as it allows for kernels being only conditionally positive definite. While this level of generality is fruitful for example in the case $X=\mathbb{R}^{d}$, we believe that it is sufficient to consider only positive definite kernels for compact spaces. Indeed, note that if $X$ is compact and separable and there is a strictly positive probability measure $\nu$ on its Borel sets, then, if we only consider kernels $k$ such that $\int_{X} k(x, y) \mathrm{d} \nu(x)$ does not depend on $y$, we know by Bochner (1941, Theorem 2) that conditionally positive definite kernels and positive definite kernels are the same up to a constant.

In the following theorem, we show propriety of the kernel score without requiring continuity of the kernel by using the theory of reproducing kernel Hilbert spaces (RKHS). In addition, we obtain a necessary and sufficient condition for the kernel score to be strictly proper.

Theorem 1.1. Let $k$ be a measurable kernel with $R K H S H$ with norm $\|\cdot\|_{H}$ and $\Phi: \mathcal{M}_{1}^{k}(X) \rightarrow H$ be the kernel embedding defined by

$$
\Phi(P):=\int k(\cdot, \omega) \mathrm{d} P(\omega) .
$$

Then the kernel score satisfies

$$
\|\Phi(P)-\Phi(Q)\|_{H}^{2}=2\left(\int S_{k}(Q, x) \mathrm{d} P(x)-\int S_{k}(P, x) \mathrm{d} P(x)\right)
$$

for all $P, Q \in \mathcal{M}_{1}^{k}(X)$. In particular, $S_{k}$ is a proper scoring rule with respect to $\mathcal{M}_{1}^{k}(X)$, and it is strictly proper if and only if the kernel embedding $\Phi$ is injective.

Note that functions of the form (1.3) are also considered in potential theory, but there $k$ is not necessary a reproducing kernel, see e.g. Landkof $(1972$, p. $58 \mathrm{ff})$ for Riesz kernels $k\left(x, x^{\prime}\right):=$ $\left|x-x^{\prime}\right|^{\alpha-d}$ defined on subsets of $\mathbb{R}^{d}$, and e.g. Fuglede (1960) and Landkof (1972, p. 363ff) for more abstract kernels. We will comment on similarities and differences between potential theory and our results in later sections.

In the machine learning literature a bounded measurable kernel is called characteristic if the kernel (mean) embedding $\Phi: \mathcal{M}_{1}(X) \rightarrow H$ defined by (1.3) is injective (Fukumizu et al., 2008; Sriperumbudur et al., 2010a). Consequently, Theorem 1.1 shows that, for bounded measurable kernels $k$, strictly proper kernel scores $S_{k}$ are exactly those for which $k$ is characteristic. In particular, the wealth of examples and conditions for characteristic kernels can be directly used to find new strictly proper scoring rules and vice versa.

While Theorem 1.1 is an interesting observation for both machine learning and probabilistic forecasting, its proof is actually rather trivial. In the rest of the paper, we therefore focus on more involved aspects of characteristic kernels, and strictly proper kernel scores, respectively. We introduce the necessary mathematical machinery on kernels and their interaction with measures in Section 2. In particular we recall that a bounded measurable kernel $k$ with RKHS $H$ induces a semi-norm $\|\cdot\|_{H}$ on $\mathcal{M}(X)$, the space of all finite signed measures on $X$, via the kernel mean embedding. In Section 3.1, we study this semi-norm for general $X$. In particular, Theorem 3.1 shows that for injective kernel embeddings, $\|\cdot\|_{H}$ fails to be equivalent to the total variation norm on $\mathcal{M}(X)$ if and only if $\operatorname{dim} \mathcal{M}(X)=\infty$, and Corollary 3.7 gives an even sharper result on $\mathcal{M}_{1}(X)$. In view of (1.4), these results show that the value on the right-hand side of (1.4) is not proportional to the (squared) total variation norm. We further present a simple computation of the left-hand side of (1.4) in terms of eigenvalues and -functions of a suitable integral operator. In Section 3.2 , we then exploit our general theory to obtain new results for bounded continuous kernels on locally compact Hausdorff spaces. For example, Theorem 3.16 and Corollary 3.17 characterizatize 
universal and characteristic kernels in terms of eigenfunctions of certain integral operators. We also provide insight concerning the difference of considering Borel or Radon measures on locally compact Hausdorff spaces in the study of kernel embeddings, see Theorems 3.12 and 3.13. As a result, it turns out in Theorem 3.14 that continuous characteristic kernels on compact Hausdorff spaces only exist if the spaces are metrizable. In Section 4, we apply the characterizations of Section 3.2 to translation-invariant kernels on compact Abelian groups and to isotropic kernels on spheres. All proofs can be found in Section 5.

\section{Preliminaries}

In this section we recall some facts about reproducing kernels and their interaction with measures. To this end, let $(X, \mathcal{A})$ be a measurable space. We denote the space of finite signed measures on $X$ by $\mathcal{M}(X)$ and write $\mathcal{M}_{+}(X)$ and $\mathcal{M}_{1}(X)$ for the subsets of all (non-negative) finite, respectively probability measures. Moreover, we write

$$
\mathcal{M}_{0}(X):=\{\mu \in \mathcal{M}(X): \mu(X)=0\} .
$$

We equip $\mathcal{M}(X)$ and its subsets above with the total variation norm $\|\cdot\|_{\mathrm{T} V}$. Recall that $\|\cdot\|_{\mathrm{T} V}$ is complete and hence $\mathcal{M}(X)$ is a Banach space. Moreover, $\mathcal{M}_{0}(X)$ is closed subspace of codimension 1 , and for every $P \in \mathcal{M}_{1}(X)$ we have

$$
\mathcal{M}(X)=\mathbb{R} P \oplus \mathcal{M}_{0}(X) .
$$

Given a measurable $f: X \rightarrow \mathbb{R}$ and a measure $\nu$ on $X$ we write $[f]_{\sim}$ for the $\nu$-equivalence class of $f$. We denote the space of $p$-times $\nu$-integrable functions by $\mathcal{L}_{p}(\nu)$ and the corresponding space of $\nu$-equivalence classes by $L_{p}(\nu)$. Note that this rather pedantic notation is useful when dealing with RKHSs since these spaces consist of functions that can be evaluated pointwise, whereas such an evaluation does not make sense for elements in $L_{p}(\nu)$ in general.

To formally introduce kernel mean embeddings, we need to recall the notion of Pettis integrals. To this end, let $H$ be a Hilbert space and $f: X \rightarrow H$ a map. Then $f$ is weakly measurable, if $\langle w, f\rangle: X \rightarrow \mathbb{R}$ is measurable for all $w \in H$. Similarly, $f$ is weakly integrable with respect to a measure $\nu$ on $(X, \mathcal{A})$, if $\langle w, f\rangle \in \mathcal{L}_{1}(\nu)$ for all $w \in H$. In this case, there exists a unique $i_{\nu}(f) \in H$, called the Pettis integral of $f$ with respect to $\nu$, such that for all $w \in H$ we have

$$
\left\langle w, i_{\nu}(f)\right\rangle=\int_{X}\langle w, f\rangle \mathrm{d} \nu
$$

see e.g. Diestel and Uhl (1977, Chapter II.3) together with the reflexivity of $H$ and the identity $H=H^{\prime}$ between $H$ and its dual $H^{\prime}$. Using the Hahn-Jordan decomposition, it is not hard to see that $i_{\mu}(f)$ can analogously be defined for finite signed measures $\mu$. In the following, we adopt the more intuitive notation $\int_{X} f \mathrm{~d} \mu:=i_{\mu}(f)$, so that the defining equation above becomes

$$
\left\langle w, \int_{X} f \mathrm{~d} \mu\right\rangle=\int_{X}\langle w, f\rangle \mathrm{d} \mu .
$$

In the case of probability measures $\mu$, we sometimes also write $\mathbb{E}_{\mu} f:=i_{\mu}(f)$. Let us now use Pettis integrals to define kernel mean embeddings. To this end, let $H$ be an RKHS over $X$ with kernel $k$ and canonical feature map $\Phi: X \rightarrow H$, that is $\Phi(x):=k(\cdot, x)$ for all $x \in X$. Then $\Phi$ is weakly measurable if and only if $\langle h, \Phi\rangle=h$ is measurable for all $h \in H$, and hence we conclude that $\Phi$ is weakly integrable with respect to some measure $\nu$ on $X$, if and only if $h \in \mathcal{L}_{1}(\nu)$ for all 
$h \in H$. By an application of the closed graph theorem, the latter is equivalent to the continuity of the map $[\cdot]_{\sim}: H \rightarrow L_{1}(\nu)$. Recall that $H$ consists of measurable functions if $k$ is measurable, see Steinwart and Christmann (2008, Lemma 4.24), and [.] $: H \rightarrow L_{1}(\nu)$ is continuous if, e.g.

$$
\int \sqrt{k(x, x)} \mathrm{d} \nu(x)<\infty
$$

see e.g. Steinwart and Christmann (2008, Theorem 4.26). Obviously, (2.3) is still sufficient for finite signed measures if one replaces $\nu$ by $|\nu|$. For a measurable kernel $k$ with RKHS $H$ we now write

$$
\mathcal{M}^{k}(X):=\left\{\mu \in \mathcal{M}(X): H \subset \mathcal{L}_{1}(\mu)\right\}
$$

and analogously we define $\mathcal{M}_{+}^{k}(X)$ and $\mathcal{M}_{1}^{k}(X)$. Obviously, $\mathcal{M}^{k}(X)$ is a vector space containing all Dirac measures and using $(2.3)$ we see that $\mathcal{M}^{k}(X)=\mathcal{M}(X)$, if $k$ is bounded. In fact, the latter is also necessary for $\mathcal{M}^{k}(X)=\mathcal{M}(X)$ as a combination of Sriperumbudur et al. (2010a, Proposition 2) with Steinwart and Christmann (2008, Lemma 4.23) shows. Moreover, our considerations above show that $\mathcal{M}^{k}(X)$ is the largest set on which we can define the kernel embedding

$$
\Phi(\mu):=\int_{X} \Phi \mathrm{d} \mu=\int_{X} k(\cdot, x) \mathrm{d} \mu(x) .
$$

For Dirac measures $\delta_{x}$ we have $\Phi\left(\delta_{x}\right)=\Phi(x)$, and since $\Phi: \mathcal{M}^{k}(X) \rightarrow H$ is linear, we see that

$$
\|\mu\|_{H}:=\|\Phi(\mu)\|_{H}
$$

defines a new semi-norm on $\mathcal{M}^{k}(X)$. By a double application of (2.2) we further have

$$
\left\langle\Phi\left(\mu_{1}\right), \Phi\left(\mu_{2}\right)\right\rangle_{H}=\int_{X} \int_{X} k\left(x, x^{\prime}\right) \mathrm{d} \mu_{1}(x) \mathrm{d} \mu_{2}\left(x^{\prime}\right) .
$$

Note that the semi-norm at (2.4) is a norm, if and only if the kernel embedding $\Phi: \mathcal{M}^{k}(X) \rightarrow H$ is injective and by (2.5) the latter is equivalent to

$$
\|\mu\|_{H}^{2}=\iint k\left(x, x^{\prime}\right) \mathrm{d} \mu(x) \mathrm{d} \mu\left(x^{\prime}\right)>0
$$

for all $\mu \in \mathcal{M}^{k}(X) \backslash\{0\}$. This leads to the following definition.

Definition 2.1. Let $k$ be a measurable kernel on $X$ and $\mathcal{M} \subset \mathcal{M}^{k}(X)$. Then $k$ is called strictly integrally positive definite with respect to $\mathcal{M}$, if (2.6) holds for all $\mu \in \mathcal{M}$ with $\mu \neq 0$.

It is well-known, that using (2.2) the semi-norm introduced above can also be computed by

$$
\|\mu\|_{H}=\left\|\int_{X} \Phi \mathrm{d} \mu\right\|_{H}=\sup _{f \in B_{H}}\left|\left\langle f, \int_{X} \Phi \mathrm{d} \mu\right\rangle\right|=\sup _{f \in B_{H}}\left|\int_{X}\langle f, \Phi\rangle \mathrm{d} \mu\right|=\sup _{f \in B_{H}}\left|\int_{X} f \mathrm{~d} \mu\right|
$$

where $B_{H}$ denotes the closed unit ball of $H$. We thus have $\|\mu\|_{H} \leq\left\|[\cdot]_{\sim}: H \rightarrow L_{1}(\mu)\right\|$, and if $k$ is bounded, then $\Phi: \mathcal{M}(X) \rightarrow H$ is continuous with $\|\Phi: \mathcal{M}(X) \rightarrow H\| \leq\|k\|_{\infty}^{1 / 2}$. In particular, if $k$ is bounded and $\Phi: \mathcal{M}(X) \rightarrow H$ is injective, then $\|\cdot\|_{H}$ defines a new norm on $\mathcal{M}(X)$ that is dominated by $\|\cdot\|_{\mathrm{T} V}$ and describes a Euclidean geometry with inner product (2.5). Unless $\operatorname{dim} \mathcal{M}(X)<\infty$, however, the two norms are not equivalent, as we will see in Theorem 3.1. 
Using the semi-norm $\|\cdot\|_{H}$ on $\mathcal{M}^{k}(X)$ we can now define a semi-metric on $\mathcal{M}_{1}^{k}(X)$ by setting

$$
\gamma_{k}(P, Q):=\|P-Q\|_{H}=\sup _{f \in B_{H}}\left|\int f \mathrm{~d} P-\int f \mathrm{~d} Q\right|
$$

for $P, Q \in \mathcal{M}_{1}^{k}(X)$. Here, we note that the second equality, which follows from (2.7), has already been shown by Sriperumbudur et al. (2010a, Theorem 1). Clearly, $k$ is characteristic, if and only if $\gamma_{k}$ is a metric, and a literal repetition of Sriperumbudur et al. (2010a, Lemma 8) shows:

Proposition 2.2. Let $(X, \mathcal{A})$ be a measurable space and $k$ a bounded measurable kernel on $X$. Then the following statements are equivalent:

i) $k$ is strictly integrally positive definite with respect to $\mathcal{M}_{0}(X)$.

ii) $k$ is characteristic.

Now, let $\nu$ be a measure on $X$ and $k$ be a measurable kernel on $X$ with RKHS $H$ such that the map $I_{k, \nu}: H \rightarrow L_{2}(\nu)$ given by $f \mapsto[f]_{\sim}$ is well-defined and compact. For examples of such $I_{k, \nu}$ recall Steinwart and Scovel (2012, Lemma 2.3), which shows that $I_{k, \nu}$ is Hilbert-Schmidt if

$$
\int_{X} k(x, x) \mathrm{d} \nu(x)<\infty
$$

Obviously, (2.8) holds, if $k$ is bounded and $\nu$ is a finite measure. Now assume that $I_{k, \nu}$ is welldefined and compact. Then, the associated integral operator $T_{k, \nu}: L_{2}(\nu) \rightarrow L_{2}(\nu)$, defined by

$$
T_{k, \nu} f=\left[\int_{X} k(x, \cdot) f(x) \mathrm{d} \nu(x)\right]_{\sim}, \quad f \in L_{2}(\nu),
$$

satisfies $T_{k, \nu}=I_{k, \nu} \circ I_{k, \nu}^{*}$, see e.g. Steinwart and Scovel (2012, Lemma 2.2), where $I_{k, \nu}^{*}$ denotes the adjoint of $I_{k, \nu}$. Therefore, $T_{k, \nu}$ is compact, positive, and self-adjoint, and if (2.8) is satisfied, $T_{k, \nu}$ is even nuclear. Moreover, the spectral theorem in the form of Steinwart and Scovel (2012, Lemma 2.12) gives us an at most countable, ordered family $\left(\lambda_{i}\right)_{i \in I} \subset(0, \infty)$ converging to 0 and a family $\left(e_{i}\right)_{i \in I} \subset H$ such that:

i) $\left(\lambda_{i}\right)_{i \in I}$ are the non-zero eigenvalues of $T_{k, \nu}$ including multiplicities,

ii) $\left(\left[e_{i}\right]_{\sim}\right)_{i \in I}$ is an $L_{2}(\nu)$-ONS of the corresponding eigenfunctions with

$$
{\overline{\operatorname{span}\left\{\left[e_{i}\right]_{\sim}: i \in I\right\}}}^{L_{2}(\nu)}={\overline{[H]_{\sim}}}^{L_{2}(\nu)},
$$

iii) $\left(\sqrt{\lambda_{i}} e_{i}\right)_{i \in I}$ is an ONS in $H$.

Here, we say that an at most countable family $\left(\alpha_{i}\right)_{i \in I} \subset[0, \infty)$ converges to 0 , if either $I=$ $\{1, \ldots, n\}$ or $I=\mathbb{N}$ and $\lim _{i \rightarrow \infty} \alpha_{i}=0$. For nuclear $T_{k, \nu}$, we additionally have $\sum_{i \in I} \lambda_{i}<\infty$, and if $k$ is bounded, $\left\|e_{i}\right\|_{\infty}<\infty$ holds for all $i \in I$. Finally, Steinwart and Scovel (2012, Theorem 3.1) show that the injectivity of $I_{k, \nu}: H \rightarrow L_{2}(\nu)$ is equivalent to either of the following statements:

i) $\left(\sqrt{\lambda_{i}} e_{i}\right)_{i \in I}$ is an ONB of $H$.

ii) For all $x, x^{\prime} \in X$ we have

$$
k\left(x, x^{\prime}\right)=\sum_{i \in I} \lambda_{i} e_{i}(x) e_{i}\left(x^{\prime}\right) .
$$


Let us now recall some notions related to measures on topological spaces, see e.g. Bauer (2001, Chapter IV) for details. To this end, let $(X, \tau)$ be a Hausdorff (topological) space and $\nu$ a measure on its Borel- $\sigma$-algebra $\mathcal{B}(X)$. Then $\nu$ is a Borel measure, if $\nu(K)<\infty$ for all compact $K \subset X$ and $\nu$ is called strictly positive, if $\nu(O)>0$ for all non-empty $O \in \tau$. Moreover, a finite measure $\nu$ on $\mathcal{B}(X)$ is a (finite) Radon measure if it is regular, i.e. if for all $B \in \mathcal{B}(X)$ we have

$$
\nu(B)=\sup \{\nu(K): K \text { compact and } K \subset B\}=\inf \{\nu(O): O \text { open and } B \subset O\} .
$$

A finite signed Radon measure is simply the difference of two finite Radon measures. In the following, we denote the space of all finite signed Radon measures by $\mathcal{M}^{*}(X)$ and the cone of (non-negative) finite Radon measures by $\mathcal{M}_{+}^{*}(X)$. As usual, $\mathcal{M}^{*}(X)$ is equipped with the norm of total variation. Obviously, every finite Radon measure is a finite Borel measure, and by Ulam's theorem, see e.g. Bauer (2001, Lemma 26.2), the converse implication is true if $X$ is a Polish space. In this respect recall that compact, metrizable spaces are Polish.

Now, let $X$ be a locally compact Hausdorff space and $C_{0}(X)$ be the space of continuous functions vanishing at infinity, equipped with the supremum norm. Then, Riesz's representation theorem, see Hewitt and Stromberg (1965, Theorem 20.48 together with Definition 20.41, Theorem 12.40, Definition 12.39, and a translation into the real-valued case by Theorem 12.36) shows that

$$
\begin{aligned}
\mathcal{M}^{*}(X) & \rightarrow C_{0}(X)^{\prime} \\
\mu & \mapsto\left(f \mapsto\langle f, \mu\rangle:=\int_{X} f \mathrm{~d} \mu\right)
\end{aligned}
$$

is an isometric isomorphism. In particular, this holds for compact $X$, and for such $X$, the space $C_{0}(X)$ coincides, of course, with the space of continuous functions $C(X)$.

Given a locally compact Hausdorff space $X$, a continuous kernel $k$ on $X$ with RKHS $H$ is called universal if $H \subset C_{0}(X)$ and $H$ is dense in $C_{0}(X)$. Note that for compact $X$ the inclusion $H \subset C_{0}(X)$ is automatically satisfied. Examples of universal kernels as well as various necessary and sufficient conditions for universality can be found in e.g. Steinwart (2001); Micchelli et al. (2006); Sriperumbudur et al. (2011); Chen et al. (2016) and the references mentioned therein.

Finally, we note that the semi-norm $\|\cdot\|_{H}$ on $\mathcal{M}^{k}(X)$ is also investigated in potential theory. There, the quantities $\|\mu\|_{H}^{2}$ and $\left\langle\mu_{1}, \mu_{2}\right\rangle_{H}$ are called energy and mutual energy, respectively, and the space $\mathcal{M}^{k}(X)$ is usually denoted by $\mathcal{E}$, see e.g. Fuglede (1960) or Landkof (1972, Chapter I, $\S 4)$. However, the foci of potential theory differ from ours. Indeed, we are mostly interested in a) investigating situations in which $\|\cdot\|_{H}$ becomes a norm and b) how this norm differs from the total variation norm. Potential theory, on the other hand, for example, investigates $a$ ) the behavior of the kernel embedding $\Phi(\mu): X \rightarrow \mathbb{R}$ for a fixed $\mu \in \mathcal{M}_{1}(X)$ by means of maximum principles or of differences of (super)-harmonic functions, see e.g. Landkof (1972, Chapter I, §3 and $\S 5)$; b) capacities and minimal energy problems e.g. of the form

$$
W(A):=\inf \left\{\|\mu\|_{H}^{2}: \mu \in \mathcal{M}_{1}^{k}(X) \text { with } \operatorname{supp} \mu \subset A\right\},
$$

see e.g. Fuglede $(1960$, p. $153 \mathrm{ff})$; and $c$ ) the relationship between the $\|\cdot\|_{H}$-topology on $\mathcal{M}_{1}^{k}(X)$, its associated weak topology, the vague topology for $X$ being a locally compact space, and the socalled fine topology see. e.g. Fuglede (1960, p. 164ff) and Landkof (1972, Chapter V, §3). Despite these differences, however, there are certainly also results from potential theory that may be interesting for characteristic kernels in machine learning and vice versa. Although a full account is out of the scope of this paper, we note that the minimal energy problem mentioned above may be interesting for better understanding how small $\|P-Q\|_{H}$ may become if $P, Q$ are probability measures with fixed disjoint supports. Two more interesting relationships will be discussed after Theorems 3.1 and 3.14 . 


\section{New Characterizations}

In this section we first compare the norms $\|\cdot\|_{H}$ and $\|\cdot\|_{\mathrm{T} V}$ and show that in infinite dimensional they are never equivalent. By establishing some structural result for characteristic kernels, we then demonstrate that characteristic kernels cannot reliably distinguish between distributions that are far away with respect to $\|\cdot\|_{\mathrm{T} V}$. We further relate the eigenfunctions of the kernel to the metric $\gamma_{k}$ and with the help of this relation we investigate continuous kernels on (locally) compact spaces. Finally, we characterize those compact spaces $X$ on which characteristic kernels exist.

\subsection{General results}

In this subsection we investigate the semi-norm $\|\cdot\|_{H}$ on $\mathcal{M}(X)$ for bounded kernels and general $X$. We begin with a result that compares $\|\cdot\|_{H}$ with $\|\cdot\|_{\mathrm{T} V}$.

Theorem 3.1. Let $(X, \mathcal{A})$ be a measurable space and $H$ the RKHS of a bounded and measurable kernel $k$ on $X$ such that the kernel embedding $\Phi: \mathcal{M}(X) \rightarrow H$ is injective. Then, the following statements are equivalent:

i) The space $\mathcal{M}(X)$ is finite dimensional.

ii) The norms $\|\cdot\|_{H}$ and $\|\cdot\|_{\mathrm{T} V}$ on $\mathcal{M}(X)$ are equivalent.

iii) The norm $\|\cdot\|_{H}$ on $\mathcal{M}(X)$ is complete.

iv) The kernel embedding $\Phi: \mathcal{M}(X) \rightarrow H$ is surjective.

Theorem 3.1 shows that for most cases of interest $\left(\mathcal{M}(X),\|\cdot\|_{H}\right)$ is not a Hilbert space. To illustrate the fourth statement of Theorem 3.1, recall that the space

$$
H_{\text {pre }}:=\left\{\Phi(\mu): \mu \in \operatorname{span}\left\{\delta_{x}: x \in X\right\}\right\}
$$

is dense in $H$, see e.g. Steinwart and Christmann (2008, Theorem 4.21). Moreover, the space $\operatorname{span}\left\{\delta_{x}: x \in X\right\}$ is, in a weak sense, dense in $\mathcal{M}(X)$, and therefore it is natural to ask whether every $f \in H$ is of the form $f=\Phi(\mu)$ for some $\mu \in \mathcal{M}(X)$. Theorem 3.1 tells us that the answer is no, unless $\operatorname{dim} \mathcal{M}(X)<\infty$. In this respect recall that it has been recently mentioned by SimonGabriel and Schölkopf (2018) that the kernel embedding $\Phi$ is, in general, not surjective. However, the authors do not provide any example, or conditions, for non-surjective $\Phi$. Two examples of non-surjective kernel embeddings $\Phi$ are provided by Pillai et al. (2007, Section 3), while Theorem 3.1 shows that actually all injective $\Phi$ fail to be surjective whenever we have $\operatorname{dim} \mathcal{M}(X)=\infty$.

It has already been noted by Fuglede $(1960$, p. 166) that there are locally compact spaces $X$ and kernels $k$ on $X$ for which $\|\cdot\|_{H}$ on $\mathcal{M}(X)$ is not complete. Again, Theorem 3.1 shows that the non-completeness is purely a consequence of the size of $\mathcal{M}(X)$ as long as $k$ is characteristic. In this respect note that for the Riesz "kernels" mentioned in the introduction, Landkof (1972, Theorem 6.1) describes the $\|\cdot\|_{H}$-completion of $\mathcal{M}_{1}(X)$. Finally note that Fuglede (1960, Lemma 3.3.1) shows that under certain circumstances $\|\cdot\|_{H}$ is actually complete on the subset $\mathcal{M}_{1}(X)$ and Fuglede (1960, Lemma 3.3.1) establishes the vague-compactness of the closed unit $\|\cdot\|_{H}$-ball of $\mathcal{M}_{1}(X)$ for continuous, strictly integrally positive definite kernels on compact spaces.

Our next goal is to show that for characteristic kernels on infinite dimensional spaces $\mathcal{M}(X)$ there always exists probability measures that have maximal $\|\cdot\|_{\mathrm{T} V}$-distance but arbitrarily small $\|\cdot\|_{H}$-distance. To this end, we need a couple of preparatory results. We begin with the following lemma that investigates the effect of $\mathbf{1}_{X} \in H$. 
Lemma 3.2. Let $(X, \mathcal{A})$ be a measurable space and $H$ the $R K H S$ of a bounded and measurable kernel $k$ on $X$. If $\mathbf{1}_{X} \in H$, then $\mathcal{M}_{0}(X)$ is $\|\cdot\|_{H}$-closed in $\mathcal{M}(X)$, and if $k$ is, in addition, characteristic, then the kernel embedding $\Phi: \mathcal{M}(X) \rightarrow H$ is injective.

The next lemma computes the $\|\cdot\|_{H}$-norm, if $H$ is an RKHS of the sum of two kernels.

Lemma 3.3. Let $(X, \mathcal{A})$ be a measurable space, and $k_{1}, k_{2}$ bounded measurable kernels on $X$ with RKHSs $H_{1}$ and $H_{2}$. Let $H$ be the RKHS of the kernel $k=k_{1}+k_{2}$. Then for all $\mu \in \mathcal{M}(X)$ we have

$$
\|\mu\|_{H}^{2}=\|\mu\|_{H_{1}}^{2}+\|\mu\|_{H_{2}}^{2} .
$$

If $k_{1}$ is characteristic or has an injective kernel embedding, then the same is thus true for $k$.

In Sriperumbudur et al. (2010a, Corollary 11) it has already been show that the sum of two bounded, continuous translation-invariant kernels on $\mathbb{R}^{d}$ is characteristic, if at least one summand is characteristic. Lemma 3.3 shows that this kind of inheritance holds in the general case.

Our next lemma considers products of kernels. In particular it shows that such products can only be characteristic if the involved factors are strictly integrally positive definite.

Lemma 3.4. Let $\left(X_{1}, \mathcal{A}_{1}\right)$ and $\left(X_{2}, \mathcal{A}_{2}\right)$ be measurable spaces and $k_{1}, k_{2}$ bounded, measurable kernels on $X_{1}$ and $X_{2}$, respectively. We denote the RKHSs of $k_{1}$ and $k_{2}$ by $H_{1}$ and $H_{2}$. Moreover, let $H$ be the RKHS of the kernel $k:=k_{1} \cdot k_{2}$ on $X_{1} \times X_{2}$. Then, for all $\mu_{1} \in \mathcal{M}\left(X_{1}\right)$ and $\mu_{2} \in \mathcal{M}\left(X_{2}\right)$ we have

$$
\left\|\mu_{1} \otimes \mu_{2}\right\|_{H}=\left\|\mu_{1}\right\|_{H_{1}} \cdot\left\|\mu_{2}\right\|_{H_{2}} .
$$

In particular, if $\operatorname{dim} \mathcal{M}\left(X_{1}\right) \geq 2$ and $\operatorname{dim} \mathcal{M}\left(X_{2}\right) \geq 2$, and $k$ is characteristic, then $k_{1}$ and $k_{2}$ are strictly integrally positive definite with respect to $\mathcal{M}\left(X_{1}\right)$ and $\mathcal{M}\left(X_{2}\right)$, respectively.

Sriperumbudur et al. (2010a, Corollary 11) shows that the product of two bounded, continuous, translation-invariant kernels on $\mathbb{R}^{d}$ is characteristic on $\mathbb{R}^{d}$ if at least one factor is characteristic. At first glance, this result seems to contradict Lemma 3.4. However, a closer look reveals that their result considers the restriction of the product to the diagonal, whereas we treat the unrestricted kernel. Later in Corollary 3.15, we will see that, on compact spaces, the product of two strictly integrally positive definite kernels gives a strictly integrally positive definite kernel on the product.

We have already seen in Theorem 3.1 that in the infinite-dimensional case the norms $\|\cdot\|_{H}$ and $\|\cdot\|_{\mathrm{T} V}$ are not equivalent on $\mathcal{M}(X)$. Intuitively, this carries over to the subspace $\mathcal{M}_{0}(X)$. The following result confirms this intuition as long as $\mathcal{M}_{0}(X)$ is a $\|\cdot\|_{H}$-closed subspace of $\mathcal{M}(X)$.

Theorem 3.5. Let $(X, \mathcal{A})$ be a measurable space with $\operatorname{dim} \mathcal{M}(X)=\infty$ and $H$ be the $R K H S$ of a bounded, measurable kernel $k$ such that the kernel embedding $\Phi: \mathcal{M}(X) \rightarrow H$ is injective. If $\mathcal{M}_{0}(X)$ is a $\|\cdot\|_{H}$-closed subspace of $\mathcal{M}(X)$, then $\|\cdot\|_{H}$ and $\|\cdot\|_{\mathrm{TV}}$ are not equivalent on $\mathcal{M}_{0}(X)$.

The non-equivalence of $\|\cdot\|_{H}$ and $\|\cdot\|_{\mathrm{T} V}$ on $\mathcal{M}_{0}(X)$ has already been observed in some particular situations. For example, Sriperumbudur et al. (2010a, Theorem 23) show that for universal kernels on compact metric spaces, $\gamma_{k}$ metrizes the weak topology (in probabilist's terminology) on $\mathcal{M}_{1}(X)$, and since for $\operatorname{dim} \mathcal{M}(X)=\infty$ this weak topology is strictly coarser than the $\|\cdot\|_{\mathrm{T} V^{-}}$ topology, we see that $\|\cdot\|_{H}$ and $\|\cdot\|_{\mathrm{T} V}$ cannot be equivalent for such kernels. In addition, the non-equivalence can also be obtained from Sriperumbudur et al. (2010a, Theorems 21 and 24) for other continuous kernels on certain metric spaces. Finally recall that $\mathcal{M}_{0}(X)$ is a $\|\cdot\|_{H}$-closed subspace of $\mathcal{M}(X)$ if $\mathbf{1}_{X} \in H$ by Lemma 3.2.

With the help of Theorem 3.5 the next result shows that characteristic kernels cannot reliably distinguish between distributions that are far away in total variation norm. 
Theorem 3.6. Let $(X, \mathcal{A})$ be a measurable space with $\operatorname{dim} \mathcal{M}(X)=\infty$ and $H$ be the $R K H S$ of a characteristic kernel $k$ on $X$. Then for all $\varepsilon>0$ there exist $Q_{1}, Q_{2} \in \mathcal{M}_{1}(X)$ such that $\left\|Q_{1}-Q_{2}\right\|_{\mathrm{T} V}=2$ and $\left\|Q_{1}-Q_{2}\right\|_{H} \leq \varepsilon$.

Theorem 3.6 only shows that there are some distributions that cannot be reliably distinguished. The following corollary shows that such distributions actually occur everywhere.

Corollary 3.7. Let $(X, \mathcal{A})$ be a measurable space with $\operatorname{dim} \mathcal{M}(X)=\infty$ and $H$ be the RKHS of a characteristic kernel $k$ on $X$. Then for all $P \in \mathcal{M}_{1}(X), \delta \in(0,2]$, and $\varepsilon \in(0, \delta)$ there exist $Q_{1}, Q_{2} \in \mathcal{M}_{1}(X)$ such that $\left\|P-Q_{i}\right\|_{\mathrm{T} V} \leq \delta$ for $i=1,2,\left\|Q_{1}-Q_{2}\right\|_{\mathrm{T} V}=\delta$, and $\left\|Q_{1}-Q_{2}\right\|_{H} \leq \varepsilon$.

The next goal of this subsection is to investigate the $\|\cdot\|_{H}$-norm with the help of the eigenvalues and eigenfunctions of the integral operator $T_{k, \nu}$. We begin with the following lemma that computes the inner product (2.5) by these eigenvalues and -functions.

Lemma 3.8. Let $(X, \mathcal{A}, \nu)$ be $\sigma$-finite measure space and $k$ be a bounded, measurable kernel with RKHS $H$ for which $I_{k, \nu}: H \rightarrow L_{2}(\nu)$ is compact and injective. Moreover, let $\left(\lambda_{i}\right)_{i \in I} \subset(0, \infty)$ and $\left(e_{i}\right)_{i \in I} \subset H$ be as around (2.10). Then, for all $\mu_{1}, \mu_{2} \in \mathcal{M}(X)$, we have

$$
\int_{X} \int_{X} k\left(x, x^{\prime}\right) \mathrm{d} \mu_{1}(x) \mathrm{d} \mu_{2}\left(x^{\prime}\right)=\sum_{i \in I} \lambda_{i} \cdot\left(\int_{X} e_{i} \mathrm{~d} \mu_{1}\right) \cdot\left(\int_{X} e_{i} \mathrm{~d} \mu_{2}\right) .
$$

For an interpretation of this lemma, we write, for bounded measurable $f: X \rightarrow \mathbb{R}$ and $\mu \in \mathcal{M}(X),\langle f, \mu\rangle=\int_{X} f \mathrm{~d} \mu$. Combining Lemma 3.8 with (2.5) we then have

$$
\left\langle\Phi\left(\mu_{1}\right), \Phi\left(\mu_{2}\right)\right\rangle_{H}=\sum_{i \in I} \lambda_{i}\left\langle e_{i}, \mu_{1}\right\rangle\left\langle e_{i}, \mu_{2}\right\rangle .
$$

In other words, all calculations regarding inner products and norms of the kernel embedding $\mu \mapsto \Phi(\mu)$ can be carried over to a weighted $\ell_{2}$-space.

To formulate the following theorem, we denote, for a $\sigma$-finite measure $\nu$ on $(X, \mathcal{A})$, the set of all $\nu$-probability densities contained in $L_{2}(\nu)$ by $\Delta(\nu)$, that is

$$
\Delta(\nu):=\left\{[h]_{\sim} \in L_{2}(\nu) \cap L_{1}(\nu):[h]_{\sim} \geq 0 \text { and } \int_{X} h \mathrm{~d} \nu=1\right\} .
$$

Moreover, we write $\mathcal{P}_{2}(\nu):=\left\{h \mathrm{~d} \nu:[h]_{\sim} \in \Delta(\nu)\right\}$ for the corresponding set of distributions.

We can now formulate the following theorem that characterizes non-characteristic kernels on $\mathcal{P}_{2}(\nu)$ and that also establishes a result similar to Theorem 3.6 for non-characteristic kernels.

Theorem 3.9. Let $(X, \mathcal{A}, \nu)$ be $\sigma$-finite measure space and $k$ a bounded, measurable kernel with RKHS $H$ for which $I_{k, \nu}: H \rightarrow L_{2}(\nu)$ is compact and injective. Then for all $[h]_{\sim},[g]_{\sim} \in \Delta(\nu)$ and $P:=h \mathrm{~d} \nu, Q:=g \mathrm{~d} \nu$ the kernel mean distance can be computed by

$$
\gamma_{k}^{2}(P, Q)=\sum_{i \in I} \lambda_{i}\left\langle[h-g]_{\sim},\left[e_{i}\right]_{\sim}\right\rangle_{L_{2}(\nu)}^{2} .
$$

Moreover, the following statements are equivalent:

i) There exist $Q_{1}, Q_{2} \in \mathcal{P}_{2}(\nu)$ with $Q_{1} \neq Q_{2}$ and $\gamma_{k}^{2}\left(Q_{1}, Q_{2}\right)=0$.

ii) There exists an $[f]_{\sim} \in L_{1}(\nu) \cap[H]_{\sim}^{\perp}$ with $[f]_{\sim} \neq 0$ and $\int_{X} f \mathrm{~d} \nu=0$.

iii) There exist $\left[h_{1}\right]_{\sim},\left[h_{2}\right]_{\sim} \in \Delta(\nu)$ with $\left[h_{1}\right]_{\sim} \neq\left[h_{2}\right]_{\sim}$ such that for all $i \in I$ we have

$$
\left\langle\left[h_{1}\right]_{\sim},\left[e_{i}\right]_{\sim}\right\rangle_{L_{2}(\nu)}=\left\langle\left[h_{2}\right]_{\sim},\left[e_{i}\right]_{\sim}\right\rangle_{L_{2}(\nu)} .
$$


Moreover, if one, and thus all, statements are true we actually find for all $P \in \mathcal{P}_{2}(\nu)$ and $\varepsilon \in(0,2)$ some $Q_{1}, Q_{2} \in \mathcal{P}_{2}(\nu)$ with $\left\|P-Q_{i}\right\|_{\mathrm{T} V} \leq \varepsilon,\left\|Q_{1}-Q_{2}\right\|_{\mathrm{T} V}=\varepsilon$, and $\gamma_{k}^{2}\left(Q_{1}, Q_{2}\right)=0$.

Equation (3.1) can also be used to show that under certain circumstances $\|\cdot\|_{H}$ cannot reliably identify, for example, the uniform distribution. The following result, which is particularly interesting in view of Section 4, illustrates this.

Corollary 3.10. Let $(X, \mathcal{A}, \nu)$ be a probability space and $k$ a bounded, measurable kernel with RKHS $H$ for which $I_{k, \nu}: H \rightarrow L_{2}(\nu)$ is compact and injective. Assume that there is one eigenfunction $e_{i_{0}}$ with $e_{i_{0}}=\mathbf{1}_{X}$. In addition assume that there are constants $c_{1}>0$ and $c_{\infty}<\infty$ with $\left\|e_{i}\right\|_{L_{1}(\nu)} \geq c_{1}$ and $\left\|e_{i}\right\|_{\infty} \leq c_{\infty}$ for all $i \in I$. For $\alpha:=c_{\infty}^{-1}$ and $j \neq i_{0}$ consider the signed measure $Q_{j}:=\left(\mathbf{1}_{X}+\alpha e_{j}\right) d \nu$. Then $Q_{j}$ is actually a probability measure and for $P:=\nu$ we have both

$$
\left\|P-Q_{j}\right\|_{\mathrm{T} V} \geq c_{1} c_{\infty}^{-1} \quad \text { and } \quad\left\|P-Q_{j}\right\|_{H}=\lambda_{j} c_{\infty}^{-2} .
$$

The last result of this subsection provides some necessary conditions for characteristic kernels.

Corollary 3.11. Let $(X, \mathcal{A}, \nu)$ be finite measure space and $k$ be a bounded, measurable kernel with RKHS $H$ for which $I_{k, \nu}: H \rightarrow L_{2}(\nu)$ is compact and injective. Then we have:

i) If $\operatorname{dim}[H]_{\sim}^{\perp} \geq 2$ in $L_{2}(\nu)$, then $k$ is not characteristic.

ii) If $\operatorname{dim}[H]_{\sim}^{\perp} \geq 1$ in $L_{2}(\nu)$ and $\mathbf{1}_{X} \in H$, then $k$ is not characteristic.

\subsection{Continuous Kernels on Locally Compact Subsets}

In this subsection, we apply the general theory developed so far to bounded continuous kernels on locally compact Hausdorff spaces $(X, \tau)$. Let us begin with some preparatory remarks. To this end, let $k$ be a bounded, continuous kernel on $X$ whose RKHS $H$ satisfies $H \subset C_{0}(X)$. In the following we call such a $k$ a $C_{0}(X)$-kernel. Our goal in this section is to investigate when $C_{0}(X)$-kernels are universal or characteristic. We begin with the following result that provides a necessary condition for the existence of strictly integrally positive definite kernels.

Theorem 3.12. Let $(X, \tau)$ be a locally compact Hausdorff space with $\mathcal{M}^{*}(X) \neq \mathcal{M}(X)$. Then no $C_{0}(X)$-kernel is strictly integrally positive definite with respect to $\mathcal{M}(X)$.

Note that Sriperumbudur et al. (2011) restrict their considerations to characteristic kernels on locally compact Polish spaces, for which we automatically have $\mathcal{M}^{*}(X)=\mathcal{M}(X)$ by Ulam's theorem, see e.g. Bauer (2001, Lemma 26.2). Some other papers, however, do not carefully distinguish between $\mathcal{M}^{*}(X)$ and $\mathcal{M}(X)$, which, at last consequence, means that their results only hold if $\mathcal{M}^{*}(X)=\mathcal{M}(X)$. Theorem 3.12 shows that this restriction is natural, and actually no restriction at all. Furthermore note that for compact spaces $X$ one can use Theorem 3.12 to show that $\mathcal{M}^{*}(X)=\mathcal{M}(X)$ is necessary for the existence of characteristic kernels. We skip such a result since later in Theorem 3.14, we will be able to show an even stronger result.

Before we formulate our next result we need a bit more preparation. To this end, let $k$ be a $C_{0}(X)$-kernel on a locally compact space $(X, \tau)$. Then we have $H \subset C_{0}(x)$ and a quick closedgraph argument shows that the corresponding inclusion operator $I: H \rightarrow C_{0}(X)$ is bounded. By the identification $C_{0}(X)^{\prime}=\mathcal{M}^{*}(X)$ in (2.11) and the simple calculation

$$
\langle I h, \mu\rangle_{C_{0}(X), \mathcal{M}^{*}(X)}=\int_{X} h \mathrm{~d} \mu=\int_{X}\langle h, k(x, \cdot)\rangle_{H} \mathrm{~d} \mu(x)=\langle h, \Phi(\mu)\rangle_{H},
$$

which holds for all $h \in H$ and $\mu \in \mathcal{M}^{*}(X)$, we further find $I^{\prime}=\Phi$, where $I^{\prime}$ is the adjoint of $I$. This observation leads to Theorem 3.13, which has already been shown for compact spaces $X$ 
by Micchelli et al. (2006, Proposition 1) and for locally compact Polish spaces by Sriperumbudur et al. (2011, Proposition 4). Although the proof of the latter paper also works on general locally compact Hausdorff spaces, we decided to add the few lines for the sake of completeness.

Theorem 3.13. Let $(X, \tau)$ be a locally compact Hausdorff space and $k$ a $C_{0}(X)$-kernel. Then the following two statements are equivalent:

i) $k$ is strictly integrally positive definite with respect to $\mathcal{M}^{*}(X)$.

ii) $k$ is universal.

With the help of Theorem 3.13 we can now show that for characteristic kernels on compact spaces $X$ it suffices to consider metrizable $X$. A similar result for universal kernels, which is included in the following theorem, has already been derived by Steinwart et al. (2006).

Theorem 3.14. For a compact Hausdorff space $(X, \tau)$ the following statements are equivalent:

i) There exists a universal kernel $k$ on $X$.

ii) There exists a continuous characteristic kernel $k$ on $X$.

iii) $X$ is metrizable, i.e. there exists a metric generating the topology $\tau$.

If one and thus all statements are true, $(X, \tau)$ is a compact Polish space and $\mathcal{M}^{*}(X)=\mathcal{M}(X)$.

Theorems 3.14 and 3.13 show that continuous, strictly integrally positive definite kernels on compact Hausdorff spaces only occur if the space if metrizable. In this case, Fuglede's notions of perfect and consistent kernels can be simplified, see Fuglede (1960, Lemma 3.3.2), and using Fuglede (1960, Theorem 3.3) we see that continuous perfect kernels on a compact Hausdorff space $X$ only exist if $X$ is metrizable.

Theorem 3.14 shows that on compact spaces we may only expect universal or characteristic kernels, if the topology is metrizable. Since in this case we have $\mathcal{M}^{*}(X)=\mathcal{M}(X)$, Theorem 3.13 and Proposition 2.2 show the well-known result that every universal kernel is characteristic. In general, the converse implication is not true, see Corollary 3.18 for a precise result, but by adding some structural requirements, both notions may coincide. The following corollary illustrates this by showing that for product kernels universal and characteristic kernels coincide.

Corollary 3.15. Let $\left(X_{1}, \tau_{1}\right)$ and $\left(X_{2}, \tau_{2}\right)$ be non-trivial compact metrizable spaces and $k_{1}, k_{2}$ continuous kernels on $X_{1}$ and $X_{2}$, respectively. For the kernel $k:=k_{1} \cdot k_{2}$ on $X_{1} \times X_{2}$ the following statements are then equivalent:

i) $k$ is universal.

ii) $k$ is characteristic.

iii) $k_{1}$ and $k_{2}$ are universal.

Our next theorem, which provides a characterization of universal kernels with the help of the eigenfunctions of the integral operator $T_{k, \nu}$, is an extension of Micchelli et al. (2006, Corollary 5) from compact to arbitrary locally compact Hausdorff spaces. Before we present it, let us first make some preparatory remarks. To this end, let $\nu$ be a strictly positive and $\sigma$-finite Borel measure on $X$. For the matter of concreteness note that if $X$ contains a dense, countable subset $\left(x_{i}\right)_{i \geq 1}$ then $\nu:=\sum_{i \geq 1} \delta_{x_{i}}$ satisfies these assumptions and therefore we always find such measures on e.g. compact metric spaces. Now, let $k$ be a bounded and continuous kernel on $X$ satisfying (2.8). Then $H$ consists of continuous functions and Steinwart and Scovel (2012, Corollary 3.5) show that (2.10) holds for all $x, x^{\prime} \in X$, and consequently, the assumptions of Lemma 3.8, Theorem 3.9 , and Corollary 3.11 are satisfied. 
With these preparations we can now formulate the following characterization of universal kernels, where we note that the equivalence between i) and ii) has essentially been shown in Sriperumbudur et al. (2010b, Proposition 12).

Theorem 3.16. Let $(X, \tau)$ be a locally compact Hausdorff space, $\nu$ a strictly positive, $\sigma$-finite Borel measure on $X$, and $k$ be a $C_{0}(X)$-kernel satisfying (2.8). In addition, let $\left(e_{i}\right)_{i \in I}$ be the eigenfunctions of $T_{k, \nu}$ in (2.10). Then the following statements are equivalent:

i) $k$ is universal.

ii) For all $\mu \in \mathcal{M}^{*}(X)$ satisfying $\int_{X} e_{i} \mathrm{~d} \mu=0$ for all $i \in I$ we have $\mu=0$.

iii) The space $\operatorname{span}\left\{e_{i}: i \in I\right\}$ is dense in $C_{0}(X)$.

If one, and thus all, statements are true and $\nu \in \mathcal{M}^{*}(X)$, then $\left(\left[e_{i}\right]_{\sim}\right)_{i \in I}$ is an ONB of $L_{2}(\nu)$.

Our next result characterizes universal and characteristic kernels on compact spaces with the help of the eigenfunctions and -values of a suitable $T_{k, \nu}$. In view of Theorem 3.14 it suffices to consider compact spaces that are Polish.

Corollary 3.17. Let $(X, \tau)$ be a compact metrizable space and $k$ a continuous kernel with RKHS $H$. Moreover, let $\left(\lambda_{i}\right)_{i \in I} \subset[0, \infty)$ be a family converging to 0 and $\left(e_{i}\right)_{i \in I} \subset C(X)$ a family such that $\operatorname{span}\left\{e_{i}: i \in I\right\}$ is dense in $C(X)$ and

$$
k\left(x, x^{\prime}\right)=\sum_{i \in I} \lambda_{i} e_{i}(x) e_{i}\left(x^{\prime}\right)
$$

holds for all $x, x^{\prime} \in X$. If there is a strictly positive, finite, and regular Borel measure $\nu$ on $X$ such that $\left(\left[e_{i}\right]_{\sim}\right)_{i \in I}$ is an ONS in $L_{2}(\nu)$, then:

i) $k$ is universal if and only if $\lambda_{i}>0$ for all $i \in I$.

ii) If $e_{i_{0}}=\mathbf{1}_{X}$ for some $i_{0} \in I$, then $k$ is characteristic if and only if $\lambda_{i}>0$ for all $i \neq i_{0}$.

iii) If $\mathbf{1}_{X} \in H$ and $e_{i} \neq \mathbf{1}_{X}$ for all $i \in I$, then $k$ is characteristic if and only if $\lambda_{i}>0$ for all $i \in I$.

Let us finally make a few remarks on the relationship between universal and characteristic kernels on compact metrizable spaces $(X, \tau)$. To this end, we first recall that for such spaces we have $\mathcal{M}^{*}(X)=\mathcal{M}(X)$, and hence Theorem 3.13 shows that universal kernels are exactly those kernels that are strictly integrally positive definite with respect to $\mathcal{M}(X)$. On the other hand, Proposition 2.2 shows that characteristic kernels are exactly those kernels that are strictly integrally positive definite with respect to $\mathcal{M}_{0}(X)$. As already mentioned above this in particular shows the well-known fact that every universal kernel is characteristic. On the other hand, both $\mathcal{M}_{0}(X) \varsubsetneqq \mathcal{M}_{0}(X)$ and a comparison of i) and ii) in Corollary 3.17 suggest that there are characteristic kernels that are not universal. So far, however, this conjecture has only be confirmed by examples, see e.g. Sriperumbudur et al. (2011, Footnote 8) or Sriperumbudur et al. (2010a, Corollary 15). The next and final result of this section shows that the conjecture is actually true for all compact metrizable spaces.

Corollary 3.18. Let $(X, \tau)$ be a compact metrizable space. Then there exists a continuous, characteristic kernel on $X$ that is not universal.

Corollary 3.18 shows that without additional assumptions a characteristic kernel is in general not universal. However, with some additional assumptions both notions may coincide. Indeed, we have already seen such an example in Corollary 3.15 and another such example is given in Lemma 5.1. More examples are summarized in Sriperumbudur et al. (2011, Figure 1). 


\section{Characteristic kernels on spaces with additional structure}

In this section, we apply the theory developed above to translation-invariant or isotropic kernels on compact Abelian groups or spheres, respectively.

\subsection{Compact Abelian Groups}

In this subsection we apply the theory developed so far to translation-invariant kernels on compact Abelian groups. Here the main difficulty lies in the fact that one traditionally considers kernels on groups that are $\mathbb{C}$-valued, while we are only interested in $\mathbb{R}$-valued kernels. Although at first glance, one may not expect any problem arising from this discrepancy, it turns out that it actually does make a difference when constructing an ONB of $L_{2}(\nu)$ with the help of characters as soon as we have more than one self-inverse character.

Our first goal is to make the statements in the above paragraph precise. To this end, let $(G,+)$ be a compact Abelian group, and $\nu$ its normalized Haar measure. As usual, we write $L_{2}(G):=L_{2}(\nu)$, and for later use recall that $\nu$ is strictly positive and regular, see e.g. Hewitt and Ross $(1963$, p. 193/4). Moreover, let $(\hat{G}, \cdot)$ be the dual group of $G$, which consists of characters $e: G \rightarrow \mathbb{T}$, where $\mathbb{T}$ denotes, as usual, the unit circle in $\mathbb{C}$, see e.g. Hewitt and Ross (1963, Chapter Six) and Folland (1995, Chapter 4.1). For notational purposes, we assume that we have another group $(I,+)$ that is isomorphic to $(\hat{G}, \cdot)$ by some mapping $i \mapsto e_{i}$. This gives $e_{i+j}=e_{i} e_{j}, e_{0}=\mathbf{1}_{G}$, and since we further have $e \bar{e}=\mathbf{1}_{G}$ for all $e \in \hat{G}$, our notation also yields $e_{-i}=\bar{e}_{i}$. In particular, we have $\operatorname{Re} e_{-i}=\operatorname{Re} e_{i}$ and $\operatorname{Im} e_{-i}=-\operatorname{Im} e_{i}$ for all $i \in I$, and for all $i \in I$ with $i=-i$ the latter equality immediately yields $\operatorname{Im} e_{i}=0$. Finally, for $i \in I$ and $x, y \in G$ we have

$$
e_{i}(-y+x)=\frac{e_{i}(x)}{e_{i}(y)}=\overline{e_{i}(y)} e_{i}(x)=e_{-i}(y) e_{i}(x),
$$

and from this we derive both, $\operatorname{Re} e_{i}(-x)=\operatorname{Re} e_{i}(x)$ and $\operatorname{Im} e_{i}(-x)=-\operatorname{Im} e_{i}(x)$, as well as

$$
\operatorname{Re} e_{i}(-y+x)=\operatorname{Re} e_{i}(x) \operatorname{Re} e_{i}(y)+\operatorname{Im} e_{i}(x) \operatorname{Im} e_{i}(y) .
$$

Note that for $i \in I$ with $i=-i$, the latter formula can be simplified using $\operatorname{Im} e_{i}=0$.

Now, it is well-know that $\left(\left[e_{i}\right]_{\sim}\right)_{i \in I}$ is an ONB of $L_{2}(G, \mathbb{C})$, see e.g. Folland (1995, Corollary 4.26) and using this fact a quick application of the Stone-Weierstrass theorem shows that $\left(e_{i}\right)_{i \in I}$ is also dense in $C(G, \mathbb{C})$. Let us construct a corresponding ONB in $L_{2}(G)$. To this end, we write $I_{0}:=\{i \in I: i=-i\}$ for the set of all self-inverse elements of $I$. Moreover, we fix a partition $I_{+} \cup I_{-}=I \backslash I_{0}$ such that $i \in I_{+}$implies $-i \in I_{-}$for all $i \in I \backslash I_{0}$. Obviously, the sets $I_{0}, I_{+}, I_{-}$ form a partition of $I$. Let us now define the family $\left(e_{i}^{*}\right)_{i \in I}$ by

$$
e_{i}^{*}:= \begin{cases}\operatorname{Re} e_{i} & \text { if } i \in I_{0} \\ \sqrt{2} \operatorname{Re} e_{i} & \text { if } i \in I_{+} \\ \sqrt{2} \operatorname{Im} e_{i} & \text { if } i \in I_{-} .\end{cases}
$$

The next result shows that $\left(e_{i}^{*}\right)_{i \in I}$ is the desired family.

Lemma 4.1. Let $(G,+)$ be a compact Abelian metric group. Then each family $\left(e_{i}^{*}\right)_{i \in I}$ given by (4.2) is an $O N B$ of $L_{2}(G)$ and $\operatorname{span}\left\{e_{i}^{*}: i \in I\right\}$ is dense in $C(G)$. Finally, we have $\left\|e_{i}^{*}\right\|_{\infty} \leq \sqrt{2}$ for all $i \in I$.

In the following, we call a kernel $k$ on an Abelian group $(G,+)$ translation invariant, if there exists a $\kappa: G \rightarrow \mathbb{R}$ such that $k\left(x, x^{\prime}\right)=\kappa\left(-x+x^{\prime}\right)$ for all $x, x^{\prime} \in G$. Clearly, $k$ is continuous, if and only if $\kappa$ is. The following lemma provides a representation of translation invariant kernels. 
Lemma 4.2. Let $(G,+)$ be a compact Abelian group, $\left(e_{i}^{*}\right)_{i \in I}$ be a family of the form (4.2), and $k: G \times G \rightarrow \mathbb{R}$ be a bounded, measurable function. Then the following statements are equivalent:

i) $k$ is a bounded, measurable, and translation invariant kernel on $G$.

ii) There exists a summable family $\left(\lambda_{i}\right)_{i \in I} \subset[0, \infty)$ such that

$$
k\left(x, x^{\prime}\right)=\sum_{\lambda_{i}>0} \lambda_{i} e_{i}^{*}(x) e_{i}^{*}\left(x^{\prime}\right)=\sum_{\lambda_{i}>0} \lambda_{i} \operatorname{Re} e_{i}\left(x-x^{\prime}\right),
$$

where the series converge absolutely for all $x, x^{\prime} \in G$ as well as uniformly in $\left(x, x^{\prime}\right)$.

If one, and thus both, statements are true, then $k$ is continuous, $\left(\lambda_{i}\right)_{i \in I}$ are all, possibly vanishing, eigenvalues of $T_{k, \nu}$, and $\left(e_{i}^{*}\right)_{i \in I}$ is an ONB of the corresponding eigenfunctions.

For an interpretation of the representation (4.3) recall that $\kappa\left(-x+x^{\prime}\right)=k\left(x, x^{\prime}\right)$ for all $x, x \in G$ and hence (4.3) gives

$$
\kappa(x)=\sum_{\lambda_{i}>0} \lambda_{i} \operatorname{Re} e_{i}(-x)=\sum_{\lambda_{i}>0} \lambda_{i} \operatorname{Re} e_{i}(x)
$$

for all $x \in G$. Consequently, the second equality in (4.3) is Bochner's theorem, see e.g. Hewitt and Ross (1970, Theorem 30.3), in the case of $\mathbb{R}$-valued kernels on compact Abelian groups. Unlike this classical theorem, however, the second equality in (4.3) also describes how the representing measure of $\kappa$ on $\hat{G}$ is given by the eigenvalues of $T_{k}$ or $T_{k}^{\mathbb{C}}$. In the following, we do not need this information, in fact, we only mentioned the second equality to provide a link to the existing theory. Instead, the first equality in (4.3), which replaces the characters of $G$ by the eigenfunctions of $T_{k}$, is more important for us, since this equality actually is the Mercer representation of $k$ in the sense of (2.10) and therefore the theory developed in the previous sections becomes applicable.

The next result is an extension of Theorem 3.14 to translation-invariant kernels on compact Abelian groups.

Theorem 4.3. For any compact Abelian group $(G,+)$, the following statements are equivalent:
i) $G$ is metrizable.
ii) $\hat{G}$ is at most countable.
iii) There exists a translation-invariant universal kernel on $G$.
iv) There exists a universal kernel on $G$.
v) There exists a translation-invariant characteristic kernel on $G$.
vi) There exists a continuous characteristic kernel on $G$.

Note that the equivalence between $i$ ) and ii) can also be shown without using translationinvariant kernels, see e.g. Morris (1979, Proposition 3) or Hewitt and Ross (1963, Theorem 24.15). Our proof, to the contrary, is solely RKHS-based.

Our next result characterizes universal and characteristic translation-invariant kernels on compact Abelian groups. In view of Theorem 4.3, it suffices to consider the metrizable case.

Corollary 4.4. Let $(G,+)$ be a compact metrizable Abelian group and $k$ be a translation-invariant kernel on $G$ with representation (4.3). Then we have:

i) $k$ is universal if and only if $\lambda_{i}>0$ for all $i \in I$.

ii) $k$ is characteristic if and only if $\lambda_{i}>0$ for all $i \neq 0$. 
Corollary 4.4 generalizes Sriperumbudur et al. (2010a, Theorem 14 and Corollary 15) from $\mathbb{T}^{d}$ to arbitrary compact metrizable Abelian groups. Moreover, recall that these authors also provide a couple of translation-invariant characteristic kernels on $\mathbb{T}$ that enjoy a closed form.

As mentioned in the beginning of this section, the major difficulty in deriving a Mercer representation (4.3) for translation-invariant kernels is the handling of self-inverse characters other than the neutral element. The simplest example of a group $G$ whose dual $\hat{G}$ contains more than one self-inverse is the quotient group $\left(\mathbb{Z}_{2},+\right)$ of $(\mathbb{Z},+)$ with its subgroup $2 \mathbb{Z}$. Indeed, besides the neutral element $e_{0}, \hat{\mathbb{Z}}_{2}$ only contains the character $e_{1}$ given by $e_{1}(0):=1$ and $e_{1}(1):=-1$. Clearly, this gives $e_{1}^{2}=e_{0}$ and thus $e_{1}$ is self-inverse. Now note that a function $k: \mathbb{Z}_{2} \times \mathbb{Z}_{2} \rightarrow \mathbb{R}$ can be uniquely described by a 2-by-2 matrix $K=\left(k\left(x, x^{\prime}\right)\right)_{x, x^{\prime} \in \mathbb{Z}_{2}}$ and a simple calculation shows that $k$ is a kernel if and only if $k(0,1)=k(1,0), k(0,0) \geq 0, k(1,1) \geq 0$, and $k(0,0) k(1,1) \geq k^{2}(0,1)$. Moreover, $k$ is translation-invariant as soon as it is constant on its diagonal, and in this case the previous conditions reduce to

$$
k(0,1)=k(1,0) \quad \text { and } \quad k(0,0)=k(1,1) \geq|k(0,1)| .
$$

Now, let $k$ be a translation-invariant kernel on $\mathbb{Z}_{2}$ and $\lambda_{0}, \lambda_{1} \geq 0$ the coefficients in (4.3). Then a simple calculation shows that the describing matrix $K$ is given by

$$
K=\left(\begin{array}{cc}
\lambda_{0}+\lambda_{1} & \lambda_{0}-\lambda_{1} \\
\lambda_{0}-\lambda_{1} & \lambda_{0}+\lambda_{1}
\end{array}\right)
$$

and therefore it is not hard to see by Corollary 4.4 that $k$ is characteristic, if and only if $k(0,0) \neq$ $k(0,1)$. Similarly, $k$ is universal, if and only if $k(0,0) \neq \pm k(0,1)$.

While this example seems to be rather trivial, it already has some important applications. For example, assume that our input space $X$ is a product space for which some components belong to a compact metrizable Abelian group, while the remaining components are only allowed to attain the values 0 and 1 . In other words, $X$ is of the form $X=G \times \mathbb{Z}_{2}^{d}$, where $G$ is a compact metrizable Abelian group and $d \geq 1$. Now, an intuitive way to construct a (translation-invariant) characteristic kernel $k$ on $X$ is to take a product $k:=k_{C} \cdot k_{D}$, where $k_{C}$ and $k_{D}$ are kernels on $G$ and $\mathbb{Z}_{2}^{d}$, respectively. By Corollary 3.15 we then know that $k$ is characteristic (or universal) if and only if both $k_{C}$ and $k_{D}$ are universal. Clearly, if $k_{D}$ is itself a product of kernels $k_{1}, \ldots, k_{d}$ then $k_{D}$ is almost automatically translation-invariant and universal. Indeed, if all $k_{i}$ satisfy (4.4) with $k_{i}(0,0) \neq \pm k_{i}(0,1)$, then each $k_{i}$ is translation-invariant and universal, and thus so is $k_{D}$. It seems fair to say that most "natural" choices of $k_{i}$ will satisfy these assumptions. On the other hand, translation-invariant universal kernels $k_{C}$ on $G$ are completely characterized by Corollary 4.4, and thus it is straightforward to characterize all translation-invariant characteristic kernels $k$ on $G \times \mathbb{Z}_{2}^{d}$ of product type $k:=k_{C} \cdot k_{D}$. However, their representation (4.3) is a bit more cumbersome. Indeed, any element $(e, \omega) \in \hat{G} \times \hat{\mathbb{Z}}_{2}^{d}=\widehat{G \times \mathbb{Z}_{2}^{d}}$ with self-inverse $e \in \hat{G}$ and arbitrary $\omega \in \mathbb{Z}_{2}^{d}$ is self-inverse, where the equality in the sense of group isomorphisms can e.g. be found in Folland (1995, Proposition 4.6). Consequently, the set $I_{0}$, which intuitively may be viewed as a small set of unusual characters, may actually be rather large.

Note that the set $\mathbb{Z}_{2}$ appears in data analysis settings whenever we have categorical variables with two possible values, which quite frequently is indeed the case. Now we have seen around (4.4) that most natural choices of kernels on $\mathbb{Z}_{2}$ are actually translation-invariant, and for these kernels the results of this subsection applies. Similarly, if we have a categorical variable with an even number $m$ of possible values that have a cyclic nature, for example hours of a day or months of a year, then $\mathbb{Z}_{m}$ can be used to describe these values, and kernels that respect the cyclic nature are translation-invariant. Clearly $m / 2$ is self-inverse in $\mathbb{Z}_{m}$, and therefore our theory again applies. 
For more on structural properties of compact Abelian groups as well as for further examples we refer to Hewitt and Ross $(1963, \S 25)$ and Hofmann and Morris (2013, Chapter 8).

\subsection{Spheres}

In this subsection we consider isotropic kernels $k$ on $\mathbb{S}^{d}$ for $d \geq 1$, that is, kernels of the form $k(x, y)=\psi(\theta(x, y))$, where $\theta(\cdot, \cdot)=\arccos \langle\cdot, \cdot\rangle$ is the geodesic distance on $\mathbb{S}^{d}$ and $\langle\cdot, \cdot\rangle$ denotes the standard scalar product on $\mathbb{R}^{d+1}$. Following Gneiting (2013), let $\Psi_{d}$ be the class of all continuous functions $\psi$ on $[0, \pi]$ such that $k(x, y)=\psi(\theta(x, y))$ is a kernel on $\mathbb{S}^{d}$, and define $\Psi_{\infty}:=\cap_{d} \Psi_{d}$. We write $\Psi_{d}^{+} \subset \Psi_{d}$ for the class of functions that induce a strictly positive definite kernel on $\mathbb{S}^{d}$, and set $\Psi_{\infty}^{+}:=\cap_{d} \Psi_{d}^{+}$. It holds that $\Psi_{d+1} \subset \Psi_{d}$ and $\Psi_{d+1}^{+} \subset \Psi_{d}^{+}$, see Gneiting (2013, Corollary 1).

The following two theorems are our main results on characteristic kernels on $\mathbb{S}^{d}$.

Theorem 4.5. Let $k$ be an isotropic kernel on $\mathbb{S}^{d}$ induced by some $\psi \in \Psi_{d+2}$ or by some $\psi \in \Psi_{d+1}^{+}$. Then the following statements are equivalent:

i) $k$ is characteristic.

ii) $k$ is universal.

iii) $k$ is strictly positive definite on $\mathbb{S}^{d}$, that is, $\psi \in \Psi_{d}^{+}$.

Theorem 4.5 shows in particular that any $\psi \in \Psi_{d+1}^{+}$induces a characteristic kernel on $\mathbb{S}^{d}$. For the practically most relevant case of $\mathbb{S}^{2}$, all the parametric families of isotropic positive definite functions in Gneiting (2013, Table 1) are in $\Psi_{3}^{+}$and thus all of them are characteristic and yield strictly proper kernel scores on $\mathbb{S}^{2}$ by Theorem 1.1 and Theorem 4.5.

Theorem 4.6. Let $k$ be an isotropic kernel on $\mathbb{S}^{d}$ induced by $\psi \in \Psi_{\infty}$. Then the following statements are equivalent:

i) $k$ is characteristic.

ii) $k$ is universal.

iii) $k$ is strictly positive definite, that is, $\psi \in \Psi_{\infty}^{+}$.

Theorem 4.6 is analogous to the result of Sriperumbudur et al. (2011, Proposition 5) for radial kernels on $\mathbb{R}^{d}$.

For the proofs of Theorems 4.5 and 4.6, we need to introduce some preliminaries. By Schoenberg (1942) the functions in $\Psi_{\infty}$ have a representation of the form

$$
\psi(\theta)=\sum_{n=0}^{\infty} b_{n}(\cos (\theta))^{n}, \quad \theta \in[0, \pi]
$$

where $\left(b_{n}\right)_{n}$ is a summable sequence of non-negative coefficients termed the $\infty$-Schoenberg sequence of $\psi$. Schoenberg (1942) also showed that the functions in $\Psi_{d}$ have a representation as

$$
\psi(\theta)=\sum_{n=0}^{\infty} b_{n, d} \frac{C_{n}^{(d-1) / 2}(\cos \theta)}{C_{n}^{(d-1) / 2}(1)}, \quad \theta \in[0, \pi],
$$

where $\left(b_{n, d}\right)_{n}$ is a summable sequence of non-negative coefficients termed the $d$-Schoenberg sequence of $\psi$, and $C_{n}^{\lambda}, \lambda>0, n \in \mathbb{N}_{0}$ are the Gegenbauer polynomials; see the Digital Library of Mathematical Functions (DLMF) $(2011,18.3 .1)$. For $\lambda=0$, we set $C_{n}^{0}(\cos \theta):=\cos (n \theta)$.

Definition 4.7. A sequence of non-negative real numbers $\left(b_{n}\right)_{n \in \mathbb{N}_{0}}$ fulfills condition $b$, if $b_{n}>0$ for infinitely many even and infinitely many odd integers. 
Remark 4.8. For $\psi \in \Psi_{\infty}$ or $\psi \in \Psi_{d}, d \geq 2$, the induced isotropic kernel is strictly positive definite if and only if its Schoenberg sequence fulfills condition b, see Menegatto (1992, 1994) and Chen et al. (2003). For $d=1$, condition b remains a necessary condition for $\psi \in \Psi_{1}^{+}$as shown by Menegatto (1995). However, it is not sufficient. A simple sufficient condition for $\psi \in \Psi_{1}^{+}$that is useful for our purposes but which is not necessary is that there is $n_{0}$ such that $b_{n, 1}>0$ for all $n \geq n_{0}$. See Menegatto et al. (2006) for a necessary and sufficient condition in the case $d=1$.

Lemma 4.9. $A \psi \in \Psi_{d+2}$ is strictly positive definite on $\mathbb{S}^{d}$, if and only if $b_{n, d}>0$ for all $n \in \mathbb{N}_{0}$.

The set $\Psi_{d}^{+} \backslash \Psi_{d+2} \subset \Psi_{d}$ is not empty and also contains elements with $b_{n, d}>0$ for all $n \geq 0$. To construct an example, take any summable sequence $\left(b_{n}\right)_{n \in \mathbb{N}_{0}}$ of positive real numbers such that $b_{2}>(d(d+3) / 2) b_{0}$. Let $\psi$ be the function with $d$-Schoenberg sequence $\left(b_{n}\right)_{n \in \mathbb{N}_{0}}$. Then $\psi \in \Psi_{d}^{+}$, fulfills $b_{n, d}>0$ for all $n \geq 0$, and, by Gneiting (2013, Corollary 4), it is not a member of $\Psi_{d+2}$.

Lemma 4.10. If $\psi \in \Psi_{d+1}^{+}$, then $b_{n, d}>0$ for all $n \geq 0$.

After these preliminary considerations concerning Schoenberg sequences, we will now show Theorem 4.5 by applying Corollary 3.17. To this end, let $\left(e_{n, j}\right)_{n \in \mathbb{N}_{0}, j=1, \ldots, N(d, n)}$ denote an orthonormal basis of spherical harmonics on $\mathbb{S}^{d}$. The polynomial $e_{n, j}$ has order $n$ and $N(d, n)=$ $\left(\begin{array}{c}n+d \\ n\end{array}\right)-\left(\begin{array}{c}n+d-2 \\ n-2\end{array}\right)$; see for example Groemer (1996, Theorem 3.1.4), where we note that he works on $\mathbb{S}^{d-1}$ while we work on $\mathbb{S}^{d}$. In particular, $e_{0,0}=1$. By Groemer (1996, Theorem 3.3.3),

$$
\frac{C_{n}^{(d-1) / 2}(\langle x, y\rangle)}{C_{n}^{(d-1) / 2}(1)}=\frac{1}{N(d, n)} \sum_{j=1}^{N(d, n)} e_{n, j}(x) e_{n, j}(y),
$$

hence any isotropic kernel on $\mathbb{S}^{d}$ induced by $\psi \in \Psi_{d}$ has a Mercer representation of the form (2.10) with $\lambda_{n, j}=b_{n, d} / N(d, n)$. Moreover, Groemer (1996, Corollary 3.2.7) shows that the space $\operatorname{span}\left\{e_{n, j}: n \in \mathbb{N}_{0}, j=1, \ldots, N(d, n)\right\}$ is dense in $C\left(\mathbb{S}^{d}\right)$.

Similar to Sriperumbudur et al. (2010a, Theorem 9) for translation invariant kernels on $\mathbb{R}^{d}$, Corollary 3.17 yields the following theorem.

Theorem 4.11. The kernel induced by $\psi \in \Psi_{d}$ is

i) universal if and only if $b_{n, d}>0$ for all $n \geq 0$.

ii) characteristic if and only if $b_{n, d}>0$ for all $n \geq 1$.

We remark that part (i) of Theorem 4.11 could alternatively be derived using Sun and Cheney (1997, Theorem 2).

Corollary 4.12. Let $k$ be a kernel on $\mathbb{S}^{d}$ induced by $\psi \in \Psi_{d}$. If $k$ is characteristic, then $\psi$ is strictly positive definite, that is $\psi \in \Psi_{d}^{+}$.

For the proof of Theorem 4.6, we need the following proposition.

Proposition 4.13. Let $k$ be a kernel on $\mathbb{S}^{d}$ induced by $\psi \in \Psi_{\infty}$. If $k$ is characteristic, then $\psi$ is strictly positive definite, that is $\psi \in \Psi_{\infty}^{+}$.

Note that Proposition 4.13 is not a direct consequence of Corollary 4.12 because we only assume that $\psi$ induces a characteristic kernel on $\mathbb{S}^{d}$ for one fixed $d$. Corollary 4.12 only gives us the weaker result that if $\psi \in \Psi_{\infty}$ induces a characteristic kernel on $\mathbb{S}^{d}$ for all $d$, then it holds that $\psi \in \Psi_{\infty}^{+}$. 


\section{Proofs}

\subsection{Proofs related to Section 1}

For the proof of Theorem 1.1 we assume that the general results on kernel mean embeddings recalled in Section 2 up to Proposition 2.2 of characteristic kernels are available. Note that these results do not involve kernel scores at all, so that there is no danger of circular reasoning.

Proof of Theorem 1.1: As explained around (2.3), the condition $P \in \mathcal{M}_{1}^{k}(X)$ ensures that $\Phi(P)$ defined by (1.3) is indeed an element of $H$. Now (1.4) follows from (2.5), namely

$$
\begin{aligned}
\|\Phi(P)-\Phi(Q)\|_{H}^{2} & =\iint k(x, y) \mathrm{d} P(x) \mathrm{d} P(y)+\iint k(x, y) \mathrm{d} Q(x) \mathrm{d} Q(y)-2 \iint k(x, y) \mathrm{d} P(x) \mathrm{d} Q(y) \\
& =2\left(\int S_{k}(P, x) \mathrm{d} Q(x)-\int S_{k}(Q, x) \mathrm{d} Q(x)\right) .
\end{aligned}
$$

The remaining assertions directly follow from (1.4).

\subsection{Proofs related to Section 3.1}

Proof of Theorem 3.1: iv $\Rightarrow$ iii). By assumption, $\Phi: \mathcal{M}(X) \rightarrow H$ is bijective and since $H$ is complete, so is $\|\cdot\|_{H}$ on $\mathcal{M}(X)$.

iii $) \Rightarrow$ ii $)$. Consider the identity map id $:\left(\mathcal{M}(X),\|\cdot\|_{\mathrm{T} V}\right) \rightarrow\left(\mathcal{M}(X),\|\cdot\|_{H}\right)$. Since we have already seen in Section 2 that

$$
\|\mu\|_{H} \leq\left\|[\cdot]_{\sim}: H \rightarrow L_{1}(\mu)\right\| \leq\|k\|_{\infty} \cdot\|\mu\|_{\mathrm{T} V}
$$

holds for all $\mu \in \mathcal{M}(X)$, the identity map is continuous. In addition, it is, of course, bijective, and since both $\left(\mathcal{M}(X),\|\cdot\|_{\mathrm{T} V}\right)$ and $\left(\mathcal{M}(X),\|\cdot\|_{H}\right)$ are complete, the open mapping theorem, see e.g. Megginson (1998, Corollary 1.6.8) shows that both norms are equivalent.

ii) $\Rightarrow i)$. By the arguments of Diestel et al. $(1995$, page $63 f)$ the space $\left(\mathcal{M}(X),\|\cdot\|_{\mathrm{T} V}\right)$ is a socalled $\mathcal{L}_{1, \lambda}$-space for all $\lambda>1$, see Diestel et al. (1995, page 60) for a definition, while the Euclidean structure of $\|\cdot\|_{H}$ on $\mathcal{M}(X)$, which is inherited from $\left(H,\|\cdot\|_{H}\right)$, shows that $\left(\mathcal{M}(X),\|\cdot\|_{H}\right)$ is an $\mathcal{L}_{2, \lambda}$-space for all $\lambda>1$. Let us now assume that $\operatorname{dim} \mathcal{M}(X)=\infty$. Then, Diestel et al. (1995, Corollary 11.7) shows that $\left(\mathcal{M}(X),\|\cdot\|_{\mathrm{T} V}\right)$ has only trivial type 1 , while $\left(\mathcal{M}(X),\|\cdot\|_{H}\right)$ has optimal type 2. However, the definition of the type of a space, see Diestel et al. (1995, page 217), immediately shows that equivalent norms always share their type, and hence $\|\cdot\|_{H}$ and $\|\cdot\|_{\mathrm{T} V}$ are not equivalent on $\mathcal{M}(X)$.

i) $\Rightarrow i v$ ). If $\mathcal{M}(X)$ is finite dimensional, the $\sigma$-algebra $\mathcal{A}$ is finite. Since $k$ is assumed to be measurable, we then see that $k$ can only attain finitely many different values, and consequently, the canonical feature map $\Phi: X \rightarrow H$ also attains only finitely many different values, say $f_{1}, \ldots, f_{m} \in H$. Using Steinwart and Christmann (2008, Theorem 4.21), we conclude that $H=$ $\operatorname{span}\left\{f_{1}, \ldots, f_{m}\right\}$. We now define $A_{i}:=\Phi^{-1}\left(\left\{f_{i}\right\}\right)$ for $i=1, \ldots, m$. By construction $A_{1}, \ldots, A_{m}$ form a partition of $X$ with $A_{i} \in \mathcal{A}$ and $A_{i} \neq \emptyset$ for all $i=1, \ldots, m$. Let us fix some $x_{i} \in A_{i}$. For the corresponding Dirac measures we then have $\Phi\left(\delta_{x_{i}}\right)=\Phi\left(x_{i}\right)=f_{i}$ and since $\Phi: \mathcal{M}(X) \rightarrow H$ is linear we find $\operatorname{span}\left\{f_{1}, \ldots, f_{m}\right\} \subset \Phi(\mathcal{M}(X))$. This shows the surjectivity of $\Phi: \mathcal{M}(X) \rightarrow H$.

Proof of Lemma 3.2: Let $\left(\mu_{n}\right) \subset \mathcal{M}_{0}(X)$ be a sequence that converges to some $\mu \in \mathcal{M}(X)$ in $\|\cdot\|_{H}$. Then we have $\mu_{n}(X)=0$ for all $n \geq 1$ and we need to show $\mu(X)=0$. The latter, however, follows from (2.7), namely

$$
|\mu(X)|=\left|\int \mathbf{1}_{X} \mathrm{~d}\left(\mu_{n}-\mu\right)\right| \leq\left\|\mathbf{1}_{X}\right\|_{H}\left\|\mu_{n}-\mu\right\|_{H} \rightarrow 0 .
$$


Let us now assume that $k$ is characteristic. Then Proposition 2.2 shows that $k$ is strictly integrally positive definite with respect to $\mathcal{M}_{0}(X)$, and hence $\|\mu\|_{H}>0$ for all $\mu \in \mathcal{M}_{0}(X) \backslash\{0\}$. Now let $\mu \in \mathcal{M}(X) \backslash \mathcal{M}_{0}(X)$ and $P$ be some probability measure on $X$. By (2.1) we then find an $\alpha \in \mathbb{R}$ and some $\mu_{0} \in \mathcal{M}_{0}(X)$ such that $\alpha P+\mu_{0}=\mu$, and since $\mu \notin \mathcal{M}_{0}(X)$ we actually have $\alpha \neq 0$. Using this decomposition, and (2.7) we now find

$$
\|\mu\|_{H}=\left\|\alpha P+\mu_{0}\right\|_{H} \geq \frac{1}{\left\|1_{X}\right\|_{H}}\left|\int \mathbf{1}_{X} \mathrm{~d}\left(\alpha P+\mu_{0}\right)\right|=\frac{|\alpha|}{\left\|1_{X}\right\|_{H}}>0
$$

and hence $\Phi: \mathcal{M}(X) \rightarrow H$ is injective, see (2.6).

Proof of Lemma 3.3: By the definition of the $\|\cdot\|_{H}$-norm and (2.5) we have

$$
\|\mu\|_{H}^{2}=\int_{X} \int_{X} k_{1}\left(x, x^{\prime}\right)+k_{2}\left(x, x^{\prime}\right) \mathrm{d} \mu(x) \mathrm{d} \mu\left(x^{\prime}\right)=\|\mu\|_{H_{1}}^{2}+\|\mu\|_{H_{2}}^{2} .
$$

Moreover, if $k_{1}$ is characteristic, then $\|\mu\|_{H_{1}}^{2}>0$ for all $\mu \in \mathcal{M}_{0}(X) \backslash\{0\}$ by Proposition 2.2 and the just established formula then yields $\|\mu\|_{H}^{2}>0$ for these $\mu$. Consequently, $k$ is characteristic. Repeating the argument for $\mathcal{M}(X)$ yields the last assertion.

Proof of Lemma 3.4: By the definition of the $\|\cdot\|_{H}$-norm and (2.5) we have

$$
\begin{aligned}
\left\|\mu_{1} \otimes \mu_{2}\right\|_{H}^{2} & =\int_{X_{1} \times X_{2}} \int_{X_{1} \times X_{2}} k_{1}\left(x_{1}, x_{1}^{\prime}\right) \cdot k_{2}\left(x_{2}, x_{2}^{\prime}\right) \mathrm{d} \mu_{1} \otimes \mu_{2}\left(x_{1}, x_{2}\right) \mathrm{d} \mu_{1} \otimes \mu_{2}\left(x_{1}^{\prime}, x_{2}^{\prime}\right) \\
& =\int_{X_{1} \times X_{1}} \int_{X_{2} \times X_{2}} k_{1}\left(x_{1}, x_{1}^{\prime}\right) \cdot k_{2}\left(x_{2}, x_{2}^{\prime}\right) \mathrm{d} \mu_{1}\left(x_{1}\right) \mathrm{d} \mu_{1}\left(x_{1}^{\prime}\right) \mathrm{d} \mu_{2}\left(x_{2}\right) \mathrm{d} \mu_{2}\left(x_{2}^{\prime}\right) \\
& =\left\|\mu_{1}\right\|_{H_{1}}^{2} \cdot\left\|\mu_{2}\right\|_{H_{2}}^{2},
\end{aligned}
$$

where we note that the application of Fubini's theorem is possible, since the kernels are bounded and the measures are finite. Now assume that $k$ is characteristic, but, say, $k_{1}$ is not strictly integrally positive definite with respect to $\mathcal{M}\left(X_{1}\right)$. Then there exists a $\mu_{1} \in \mathcal{M}\left(X_{1}\right)$ with $\mu_{1} \neq 0$ but $\left\|\mu_{1}\right\|_{H_{1}}=0$. Moreover, since $\operatorname{dim} \mathcal{M}\left(X_{2}\right) \geq 2$, the decomposition (2.1) gives a $\mu_{2} \in \mathcal{M}_{0}\left(X_{2}\right)$ with $\mu_{2} \neq 0$. Let us define $\mu:=\mu_{1} \otimes \mu_{2}$. Our construction then yields $\mu \neq 0$ and $\mu\left(X_{1} \times X_{2}\right)=$ $\mu_{1}\left(X_{1}\right) \cdot \mu_{2}\left(X_{2}\right)=0$, that is $\mu \in \mathcal{M}_{0}\left(X_{1} \times X_{2}\right)$, while the already established product formula shows $\|\mu\|_{H}=\left\|\mu_{1}\right\|_{H_{1}} \cdot\left\|\mu_{2}\right\|_{H_{2}}=0$. By Proposition 2.2 we conclude that $k$ is not characteristic.

Proof of Theorem 3.5: For some fixed $P \in \mathcal{M}_{1}(X)$ we consider the map

$$
\begin{aligned}
\pi: \mathbb{R} P \oplus \mathcal{M}_{0}(X) & \rightarrow \mathcal{M}(X) \\
\alpha P+\mu_{0} & \mapsto \mu_{0} .
\end{aligned}
$$

By (2.1), $\pi$ is a linear map $\pi: \mathcal{M}(X) \rightarrow \mathcal{M}(X)$ with $\pi^{2}=\pi$ and $\operatorname{ran} \pi=\mathcal{M}_{0}(X)$, and hence a projection onto $\mathcal{M}_{0}(X)$. Moreover, $\mathcal{M}_{0}(X)$ is $\|\cdot\|_{H}$-closed by assumption and therefore $\pi$ is $\|\cdot\|_{H}$-continuous, see Megginson (1998, Theorem 3.2.14). Let us now assume that $\|\cdot\|_{H}$ and $\|\cdot\|_{\mathrm{T} V}$ were equivalent on $\mathcal{M}_{0}(X)$. Our goal is to show that this assumption implies the equivalence of $\|\cdot\|_{H}$ and $\|\cdot\|_{\mathrm{T} V}$ on $\mathcal{M}(X)$. To this end we fix some sequence $\left(\mu_{n}\right) \in \mathcal{M}(X)$ and some $\mu \in \mathcal{M}(X)$ with $\left\|\mu_{n}-\mu\right\|_{H} \rightarrow 0$. By (2.1) we then find $\alpha_{n}, \alpha \in R$ with $\mu_{n}=\alpha_{n} P+\pi\left(\mu_{n}\right)$ and $\mu=\alpha P+\pi(\mu)$. Using the $\|\cdot\|_{H}$-continuity of $\pi$, we then find $\left\|\pi\left(\mu_{n}\right)-\pi(\mu)\right\|_{H} \rightarrow 0$, and since we assumed that $\|\cdot\|_{H}$ and $\|\cdot\|_{\mathrm{T} V}$ are equivalent on $\mathcal{M}_{0}(X)$, we conclude that $\left\|\pi\left(\mu_{n}\right)-\pi(\mu)\right\|_{\mathrm{T} V} \rightarrow 0$. In addition, we have $\|P\|_{H}>0$ by the injectivity of the kernel embedding, and therefore

$$
\left|\alpha_{n}-\alpha\right| \cdot\|P\|_{H} \leq\left\|\mu_{n}-\mu\right\|_{H}+\left\|\pi\left(\mu_{n}\right)-\pi(\mu)\right\|_{H} \rightarrow 0
$$


shows that $\alpha_{n} \rightarrow \alpha$. Combining these considerations, we find

$$
\left\|\mu_{n}-\mu\right\|_{\mathrm{T} V} \leq\left|\alpha_{n}-\alpha\right| \cdot\|P\|_{\mathrm{T} V}+\left\|\pi\left(\mu_{n}\right)-\pi(\mu)\right\|_{\mathrm{T} V} \rightarrow 0 .
$$

Summing up, we have seen that $\left\|\mu_{n}-\mu\right\|_{H} \rightarrow 0$ implies $\left\|\mu_{n}-\mu\right\|_{\mathrm{T} V} \rightarrow 0$ for every sequence $\left(\mu_{n}\right) \in \mathcal{M}(X)$, and consequently, the identity map id : $\left(\mathcal{M}(X),\|\cdot\|_{H}\right) \rightarrow\left(\mathcal{M}(X),\|\cdot\|_{\mathrm{T} V}\right)$ is continuous. This yields $\|\cdot\|_{\mathrm{T} V} \leq\|\mathrm{id}\| \cdot\|\cdot\|_{H}$ on $\mathcal{M}(X)$, and since we also have $\|\cdot\|_{H} \leq\|k\|_{\infty} \cdot\|\cdot\|_{\mathrm{T} V}$ we see that $\|\cdot\|_{H}$ and $\|\cdot\|_{\mathrm{T} V}$ are indeed equivalent on $\mathcal{M}(X)$. This, however, contradicts Theorem 3.1 , and hence our assumption that $\|\cdot\|_{H}$ and $\|\cdot\|_{\mathrm{T} V}$ were equivalent on $\mathcal{M}_{0}(X)$ is false.

Proof of Theorem 3.6: Let us first assume that $\mathbf{1}_{X} \in H$. By Lemma 3.2 we then see that the kernel embedding $\Phi: \mathcal{M}(X) \rightarrow H$ is injective, and Theorem 3.5 thus shows that $\|\cdot\|_{H}$ and $\|\cdot\|_{\mathrm{T} V}$ are not equivalent on $\mathcal{M}_{0}(X)$. Since $\|\cdot\|_{H}$ is dominated by $\|\cdot\|_{\mathrm{T} V}$, we consequently find a sequence $\left(\mu_{n}\right) \subset \mathcal{M}_{0}(X)$ and some $\delta>0$ such that $\left\|\mu_{n}\right\|_{H} \rightarrow 0$ and $\inf _{n \geq 1}\left\|\mu_{n}\right\|_{\mathrm{T} V} \geq \delta$. Let us consider the Hahn-Jordan decomposition $\mu_{n}=\mu_{n}^{+}-\mu_{n}^{-}$with $\mu_{n}^{+}, \mu_{n}^{-} \in \mathcal{M}_{+}(X)$. Since $\mu_{n} \in \mathcal{M}_{0}(X)$, we have $\mu_{n}^{+}(X)=\mu_{n}^{-}(X)$. We define

$$
Q_{n}^{(1)}:=\frac{\mu_{n}^{+}}{\mu_{n}^{+}(X)} \quad \text { and } \quad Q_{n}^{(2)}:=\frac{\mu_{n}^{-}}{\mu_{n}^{+}(X)} .
$$

Clearly, this yields $Q_{n}^{(1)}, Q_{n}^{(2)} \in \mathcal{M}_{1}(X)$ and

$$
\left\|Q_{n}^{(1)}-Q_{n}^{(2)}\right\|_{\mathrm{T} V}=\frac{\mu_{n}^{+}(X)+\mu_{n}^{-}(X)}{\mu_{n}^{+}(X)}=2 .
$$

Moreover, we have

$$
\left\|Q_{n}^{(1)}-Q_{n}^{(2)}\right\|_{H}=\frac{\left\|\mu_{n}\right\|_{H}}{\mu_{n}^{+}(X)}=\frac{\left\|\mu_{n}\right\|_{H}}{\frac{1}{2}\left\|\mu_{n}\right\|_{\mathrm{T} V}} \leq 2 \delta^{-1}\left\|\mu_{n}\right\|_{H}
$$

and by choosing $n$ sufficiently large we can therefore guarantee $\left\|Q_{n}^{(1)}-Q_{n}^{(2)}\right\|_{H} \leq \varepsilon$.

Let us now consider the case $\mathbf{1}_{X} \notin H$. By Lemma 3.3 we then see that the kernel $\tilde{k}:=k+1$ is characteristic. Let us write $H_{1}:=\mathbb{R} \mathbf{1}_{X}$ for the RKHS of the constant kernel $k_{1}:=\mathbf{1}_{X \times X}$. Then $x \mapsto\left(k(x, \cdot), k_{1}(x, \cdot)\right) \in H \times H_{1}$ is a feature map of the kernel $k+1$ in the sense of Steinwart and Christmann (2008, Definition 4.1) if $H \times H_{1}$ is equipped with the usual Hilbert space norm $\left\|\left(w, w_{1}\right)\right\|:=\sqrt{\|w\|_{H}^{2}+\left\|w_{1}\right\|_{H_{1}}^{2}}$ and consequently an application of Steinwart and Christmann (2008, Theorem 4.21) shows that the RKHS $\tilde{H}$ of $k+1$ is given by $\tilde{H}=H+H_{1}$. The latter yields $\mathbf{1}_{X} \in \tilde{H}$. The already considered case thus shows that for all $\varepsilon>0$ there exist distributions $Q_{1}, Q_{2} \in \mathcal{M}_{1}(X)$ such that $\left\|Q_{1}-Q_{2}\right\|_{\mathrm{T} V}=2$ and $\left\|Q_{1}-Q_{2}\right\|_{\tilde{H}} \leq \varepsilon$. Moreover, for $\mu:=Q_{1}-Q_{2} \in \mathcal{M}_{0}(X)$ we see by Lemma 3.3 and (2.5) that

$$
\varepsilon^{2} \geq\left\|Q_{1}-Q_{2}\right\|_{\tilde{H}}^{2}=\|\mu\|_{\tilde{H}}^{2}=\|\mu\|_{H}^{2}+\iint \mathbf{1}_{X \times X} \mathrm{~d} \mu \mathrm{d} \mu=\|\mu\|_{H}^{2}+\mu(X) \cdot \mu(X)=\|\mu\|_{H}^{2}=\left\|Q_{1}-Q_{2}\right\|_{H}^{2}
$$

we have shown the assertion in the second case, too.

Proof of Corollary 3.7: Let us fix some $P \in \mathcal{M}_{1}(X), \delta \in(0,1]$ and $\varepsilon \in(0,2 \delta)$. By Theorem 3.6 there then exist distributions $\tilde{Q}_{1}, \tilde{Q}_{2} \in \mathcal{M}_{1}(X)$ with $\left\|\tilde{Q}_{1}-\tilde{Q}_{2}\right\|_{\mathrm{T} V}=2$ and $\left\|\tilde{Q}_{1}-\tilde{Q}_{2}\right\|_{H} \leq \varepsilon$. We define $Q_{i}:=(1-\delta) P+\delta \tilde{Q}_{i} \in \mathcal{M}_{1}(X)$ for $i=1,2$. A simple calculation then shows that

$$
\left\|Q_{1}-Q_{2}\right\|_{\mathrm{T} V}=\delta\left\|\tilde{Q}_{1}-\tilde{Q}_{2}\right\|_{\mathrm{T} V}=2 \delta,
$$


and analogously, we find $\left\|Q_{1}-Q_{2}\right\|_{H} \leq \delta \varepsilon \leq \varepsilon$. Finally, we have $\left\|P-Q_{i}\right\|_{\mathrm{T} V}=\delta\left\|P-\tilde{Q}_{i}\right\|_{\mathrm{T} V} \leq 2 \delta$, and hence we obtain the assertion for $\tilde{\delta}:=2 \delta$.

Proof of Lemma 3.8: Let us first assume that $\mu_{1}, \mu_{2} \in \mathcal{M}_{+}(X)$. For $x, x^{\prime} \in X$ we define

$$
\tilde{k}\left(x, x^{\prime}\right):=\sum_{i \in I} \lambda_{i}\left|e_{i}(x)\right| \cdot\left|e_{i}\left(x^{\prime}\right)\right| .
$$

Then the Cauchy-Schwarz inequality together with (2.10) gives

$$
\left|\tilde{k}\left(x, x^{\prime}\right)\right|=\sum_{i \in I} \lambda_{i}\left|e_{i}(x)\right| \cdot\left|e_{i}\left(x^{\prime}\right)\right| \leq \sum_{i \in I} \lambda_{i} e_{i}^{2}(x) \cdot \sum_{i \in I} \lambda_{i} e_{i}^{2}\left(x^{\prime}\right)=k(x, x) \cdot k\left(x^{\prime}, x^{\prime}\right)
$$

for all $x, x^{\prime} \in X$, and since $k$ is bounded, we conclude that $\tilde{k}$ is also bounded, and hence $\tilde{k} \in$ $L_{2}\left(\mu_{1} \otimes \mu_{2}\right)$. Moreover, for finite $J \subset I$ and $x, x^{\prime} \in X$ we have

$$
\left|\sum_{i \in J} \lambda_{i} e_{i}(x) e_{i}\left(x^{\prime}\right)\right| \leq \tilde{k}\left(x, x^{\prime}\right) .
$$

Using Fubini's theorem, (2.10), Lebesgue's dominated convergence theorem, and yet another time Fubini's theorem, we thus find

$$
\begin{aligned}
\int_{X} \int_{X} k\left(x, x^{\prime}\right) \mathrm{d} \mu_{1}(x) \mathrm{d} \mu_{2}\left(x^{\prime}\right) & =\int_{X \times X} \sum_{i \in I} \lambda_{i} e_{i}(x) e_{i}\left(x^{\prime}\right) \mathrm{d}\left(\mu_{1} \otimes \mu_{2}\right)\left(x, x^{\prime}\right) \\
& =\sum_{i \in I} \int_{X \times X} \lambda_{i} e_{i}(x) e_{i}\left(x^{\prime}\right) \mathrm{d}\left(\mu_{1} \otimes \mu_{2}\right)\left(x, x^{\prime}\right) \\
& =\sum_{i \in I} \int_{X} \int_{X} \lambda_{i} e_{i}(x) e_{i}\left(x^{\prime}\right) \mathrm{d} \mu_{1}(x) \mathrm{d} \mu_{2}\left(x^{\prime}\right) .
\end{aligned}
$$

From the latter the assertion immediately follows.

Finally, let us assume that $\mu_{1}, \mu_{2} \in \mathcal{M}(X)$. Using the Hahn decompositions $\mu_{1}=\mu_{1}^{+}-\mu_{1}^{-}$, $\mu_{2}=\mu_{2}^{+}-\mu_{2}^{-}$, and the fact that the expressions on the left and right of the desired equation are linear in the involved measures, we then obtain the assertion by the already established case.

Proof of Theorem 3.9: Clearly, the signed measure $\mu:=P-Q$ has the $\nu$-density $h-g$ and we have $[h-g]_{\sim} \in L_{2}(\nu) \cap L_{1}(\nu)$. Now we find (3.1) by (2.5) and Lemma 3.8, namely

$$
\gamma_{k}^{2}(P, Q)=\int_{X} \int_{X} k\left(x, x^{\prime}\right) \mathrm{d} \mu(x) \mathrm{d} \mu\left(x^{\prime}\right)=\sum_{i \in I} \lambda_{i} \cdot\left(\int_{X} e_{i} \mathrm{~d} \mu\right)^{2}=\sum_{i \in I} \lambda_{i} \cdot\left(\int_{X} e_{i} \cdot(h-g) \mathrm{d} \nu\right)^{2} .
$$

ii) $\Rightarrow$ i). We split $f$ into $f=f^{+}-f^{-}$with $f^{+} \geq 0$ and $f^{-} \geq 0$. By our assumption we then know that

$$
c:=\int_{X} f^{+} \mathrm{d} \nu=\int_{X} f^{-} \mathrm{d} \nu>0
$$

For $\delta>0, h \in \Delta(\nu)$ and $P:=h \mathrm{~d} \nu \in \mathcal{P}_{2}(\nu)$ we define $h_{1}:=(1+\delta c)^{-1}\left(h+\delta f^{+}\right)$and $h_{2}:=$ $(1+\delta c)^{-1}\left(h+\delta f^{-}\right)$and consider the corresponding measures $Q_{1}:=h_{1} \mathrm{~d} \nu$ and $Q_{2}:=h_{2} \mathrm{~d} \nu$. The construction immediately ensures $Q_{1}, Q_{2} \in \mathcal{P}_{2}(\nu)$. Moreover, we have

$$
\begin{aligned}
\left\|P-Q_{1}\right\|_{\mathrm{T} V}=\int_{X}\left|h-h_{1}\right| \mathrm{d} \nu=\int_{X}\left|\frac{h+\delta c h}{1+\delta c}-\frac{h+\delta f^{+}}{1+\delta c}\right| \mathrm{d} \nu & =\int_{X}\left|\frac{\delta c h-\delta f^{+}}{1+\delta c}\right| \mathrm{d} \nu \\
& \leq \frac{\delta}{1+\delta c} \int_{X}|c h|+\left|f^{+}\right| \mathrm{d} \nu \\
& =\frac{2 \delta c}{1+\delta c},
\end{aligned}
$$


and analogously we find $\left\|P-Q_{2}\right\|_{\mathrm{T} V} \leq 2 \delta c /(1+\delta c)$. In addition, we have

$$
\left\|Q_{1}-Q_{2}\right\|_{\mathrm{T} V}=\frac{1}{1+\delta c} \int_{X}\left|\delta f^{+}-\delta f^{-}\right| \mathrm{d} \nu=\frac{\delta}{1+\delta c} \int_{X}|f| \mathrm{d} \nu=\frac{2 \delta c}{1+\delta c}
$$

and by using that $\{2 \delta c /(1+\delta c): \delta>0\}=(0,2)$ we obtain the norm conditions for $Q_{1}$ and $Q_{2}$. Finally, (3.1) yields

$$
\gamma_{k}^{2}\left(Q_{1}, Q_{2}\right)=\sum_{i \in I} \lambda_{i}\left\langle\left[h_{1}-h_{2}\right]_{\sim},\left[e_{i}\right]_{\sim}\right\rangle_{L_{2}(\nu)}^{2}=\frac{\delta}{1+\delta c} \sum_{i \in I} \lambda_{i}\left\langle[f]_{\sim},\left[e_{i}\right]_{\sim}\right\rangle_{L_{2}(\nu)}^{2}=0,
$$

which shows $i$ ) as well as the final assertion.

i) $\Rightarrow$ iii). Let $Q_{1}$ and $Q_{2}$ be according to $\left.i\right)$ and $\left[h_{1}\right]_{\sim},\left[h_{2}\right]_{\sim} \in \Delta(\nu)$ their $\nu$-densities. Then $Q_{1} \neq Q_{2}$ implies $\left[h_{1}\right]_{\sim} \neq\left[h_{2}\right]_{\sim}$, and (3.1) yields

$$
0=\gamma_{k}^{2}(P, Q)=\sum_{i \in I} \lambda_{i}\left\langle\left[h_{1}-h_{2}\right]_{\sim},\left[e_{i}\right]_{\sim}\right\rangle_{L_{2}(\nu)}^{2}
$$

Since $\lambda_{i}>0$ for all $i \in I$, we then conclude that $\left\langle\left[h_{1}-h_{2}\right]_{\sim},\left[e_{i}\right]_{\sim}\right\rangle_{L_{2}(\nu)}^{2}=0$ for all $i \in I$, which in turn implies iii).

iii) $\Rightarrow$ ii). We define $f:=h_{1}-h_{2}$. Clearly, we have $[f]_{\sim} \in L_{2}(\nu) \cap L_{1}(\nu)$ and $f \neq 0$. Moreover, $h_{1}, h_{2} \in \Delta(\nu)$ gives

$$
\int_{X} f \mathrm{~d} \nu=\int_{X} h_{1} \mathrm{~d} \nu-\int_{X} h_{2} \mathrm{~d} \nu=1-1=0 .
$$

Finally, the equality $\left\langle\left[h_{1}\right]_{\sim},\left[e_{i}\right]_{\sim}\right\rangle_{L_{2}(\nu)}=\left\langle\left[h_{2}\right]_{\sim},\left[e_{i}\right]_{\sim}\right\rangle_{L_{2}(\nu)}$, which holds for all $i \in I$, implies $\left\langle[f]_{\sim},\left[e_{i}\right]_{\sim}\right\rangle_{L_{2}(\nu)}=0$ for all $i \in I$, and hence $[f]_{\sim} \in[H]_{\sim}^{\perp}$.

Proof of Corollary 3.10: Let $h_{j}:=\left(\mathbf{1}_{X}+\alpha e_{j}\right)$ be the $\nu$-density of $Q_{j}$. Using $\left\|e_{j}\right\|_{\infty} \leq c_{\infty}$ and $\alpha=c_{\infty}^{-1}$, we then find $h_{j} \geq 0$, and since $\left[e_{i_{0}}\right]_{\sim} \perp\left[e_{j}\right]_{\sim}$ we further find

$$
\int_{X} h_{j} \mathrm{~d} \nu=1+\alpha \int_{X} e_{j} e_{i_{0}} \mathrm{~d} \nu=1 .
$$

Consequently, $Q_{j}$ is a probability measure. Moreover, we find

$$
\left\|P-Q_{j}\right\|_{\mathrm{T} V}=\int_{X}\left|\mathbf{1}_{X}-h_{j}\right| \mathrm{d} \nu=\int_{X}\left|\alpha e_{j}\right| \mathrm{d} \nu \geq c_{1} c_{\infty}^{-1}
$$

and (3.1) implies

$$
\left\|P-Q_{j}\right\|_{H}^{2}=\sum_{i \in I} \lambda_{i}\left\langle\left[\mathbf{1}_{X}-h_{j}\right]_{\sim},\left[e_{i}\right]_{\sim}\right\rangle_{L_{2}(\nu)}^{2}=\sum_{i \in I} \lambda_{i}\left\langle\left[-\alpha e_{j}\right]_{\sim},\left[e_{i}\right]_{\sim}\right\rangle_{L_{2}(\nu)}^{2}=\lambda_{j} c_{\infty}^{-2} .
$$

This shows the assertion.

Proof of Corollary 3.11: In both cases it suffices to show ii) of Theorem 3.9.

i). Since $\operatorname{dim}[H]_{\sim}^{\perp} \geq 2$ there exist linearly independent $\left[f_{1}\right]_{\sim},\left[f_{2}\right]_{\sim} \in[H]_{\sim}^{\perp}$. If $\int_{X} f_{2} \mathrm{~d} \nu=0$ then there is nothing left to prove, and if $\int_{X} f_{2} \mathrm{~d} \nu \neq 0$ then the desired function is

$$
f:=f_{1}-\frac{\int_{X} f_{1} \mathrm{~d} \nu}{\int_{X} f_{2} \mathrm{~d} \nu} \cdot f_{2}
$$

ii). Since $\operatorname{dim}[H]_{\sim}^{\perp} \geq 1$, there exists an $[f]_{\sim} \in[H]_{\sim}^{\perp} \backslash\{0\}$ and from $\mathbf{1}_{X} \in H$ we conclude that $\int_{X} f \mathrm{~d} \nu=\left\langle[f]_{\sim},\left[\mathbf{1}_{X}\right]_{\sim}\right\rangle_{L_{2}(\nu)}=0$. 


\subsection{Proofs and auxiliary results related to Section 3.2}

The following lemma compares strictly integrally positive definite kernels with respect to $\mathcal{M}(X)$ and $\mathcal{M}_{0}(X)$. In an implicit form, it has already been used, and a similar statement is SimonGabriel and Schölkopf (2018, Theorem 32).

Lemma 5.1. Let $(X, \mathcal{A})$ be a measurable space, and $k$ be a bounded measurable kernel on $X$. Moreover, let $\mathcal{M} \subset \mathcal{M}(X)$ be a subspace with $\mathcal{M} \cap \mathcal{M}_{1}(X) \neq \emptyset$ and $\mathcal{M}_{0}:=\mathcal{M} \cap \mathcal{M}_{0}(X)$. Then the following statements are equivalent:

i) $k$ is strictly integrally positive definite with respect to $\mathcal{M}_{0}$.

ii) $k+1$ is strictly integrally positive definite with respect to $\mathcal{M}$.

iii) $k+1$ is strictly integrally positive definite with respect to $\mathcal{M}_{0}$.

Proof. Let $\Phi_{1}$ denote the canonical feature map of the kernel $k_{1}=\mathbf{1}_{X \times X}$ and $H_{1}$ its RKHS. Moreover, we write $H+1$ for the RKHS of $k+1$. For later use, we note that (2.5) implies $\left\langle\Phi_{1}(\mu), \Phi_{1}\left(\mu_{0}\right)\right\rangle_{H_{1}}=0$ for all $\mu \in \mathcal{M}(X)$ and $\mu_{0} \in \mathcal{M}_{0}(X)$.

iii) $\Rightarrow$ ii). Let us fix a $P \in \mathcal{M} \cap \mathcal{M}_{1}(X)$. Similar to (2.1) we then have $\mathcal{M}=\mathbb{R} P \oplus \mathcal{M}_{0}$. Indeed, " $\supset$ " and $\mathbb{R} P \cap \mathcal{M}_{0}=\{0\}$ are trivial and for $\mu \in \mathcal{M}$ it is easy to see that $\mu-\mu(X) P \in \mathcal{M}_{0}$. Now, we need to prove $\|\mu\|_{H+1}>0$ for all $\mu=\alpha P+\mu_{0} \in \mathbb{R} P \oplus \mathcal{M}_{0}$ with $\mu \neq 0$. By iii) we already know this in the case $\alpha=0$, and thus we further assume $\alpha \neq 0$. Then Lemma 3.3 and (2.5) together with our initial remark yield

$$
\begin{aligned}
\|\mu\|_{H+1}^{2}=\|\mu\|_{H}^{2}+\left\langle\Phi_{1}(\mu), \Phi_{1}(\mu)\right\rangle_{H_{1}} & =\|\mu\|_{H}^{2}+\alpha^{2}\|P\|_{H_{1}}^{2}+2 \alpha\left\langle\Phi_{1}(P), \Phi_{1}\left(\mu_{0}\right)\right\rangle_{H_{1}}+\left\|\mu_{0}\right\|_{H_{1}}^{2} \\
& =\|\mu\|_{H}^{2}+\alpha^{2}
\end{aligned}
$$

and since $\alpha \neq 0$, we conclude $\|\mu\|_{H+1}^{2}>0$.

ii) $\Rightarrow$ iii). This is trivial.

iii) $\Leftrightarrow$ i). For $\mu_{0} \in \mathcal{M}_{0} \subset \mathcal{M}_{0}(X)$, Lemma 3.3 together with our initial remark shows $\left\|\mu_{0}\right\|_{H+1}^{2}=\left\|\mu_{0}\right\|_{H}^{2}+\left\|\mu_{0}\right\|_{H_{1}}^{2}=\left\|\mu_{0}\right\|_{H}^{2}$. From this equality the equivalence immediately follows.

Proof of Theorem 3.12: We fix a $C_{0}(X)$-kernel $k$ and a finite signed measure $\mu \in \mathcal{M}(X) \backslash$ $\mathcal{M}^{*}(X)$. Then $f \mapsto \int_{X} f \mathrm{~d} \mu$ defines a bounded linear operator $C_{0}(X) \rightarrow \mathbb{R}$, and by (2.11) there thus exists a $\mu^{*} \in \mathcal{M}^{*}(X)$ such that

$$
\int_{X} f \mathrm{~d} \mu=\int_{X} f \mathrm{~d} \mu^{*}
$$

for all $f \in C_{0}(X)$. By $H \subset C_{0}(X)$ and (2.7) we conclude that $\left\|\mu-\mu^{*}\right\|_{H}=0$ while our construction ensures $\mu \neq \mu^{*}$.

Proof of Theorem 3.13: Using the already observed identity $I^{\prime}=\Phi$ and Megginson (1998, Theorem 3.1.17) we see that $I$ has a dense image if and only if $\Phi: \mathcal{M}^{*}(X) \rightarrow H$ is injective. By (2.6) we then conclude that $k$ is universal if and only if $k$ is strictly integrally positive definite with respect to $\mathcal{M}^{*}(X)$.

Proof of Theorem 3.14: i) $\Leftrightarrow$ iii). This has been shown in Steinwart et al. (2006, Theorem 2 ). Moreover, it is well-known that compact metrizable Hausdorff spaces are Polish. The equality $\mathcal{M}^{*}(X)=\mathcal{M}(X)$ then follows from Ulam's theorem, see e.g. Bauer (2001, Lemma 26.2).

i) $\Rightarrow$ ii). If there exists a universal kernel $k$, then we have already shown that $\mathcal{M}^{*}(X)=\mathcal{M}(X)$. Consequently, $k$ is strictly integrally positive definite with respect to $\mathcal{M}(X)$ by Theorem 3.13 , and thus characteristic by Proposition 2.2. 
ii) $\Rightarrow$ i). Assume that there exists a characteristic kernel $k$. By Proposition 2.2 we know that $k$ is strictly integrally positive definite with respect to $\mathcal{M}_{0}(X)$. Then $k+1$ is a bounded and continuous kernel, which is strictly integrally positive definite with respect to $\mathcal{M}(X)$ by Lemma 5.1. Using $\mathcal{M}^{*}(X) \subset \mathcal{M}(X)$ and Theorem 3.13 we conclude that $k$ is universal.

Proof of Corollary 3.15: $i) \Rightarrow$ ii). Since $\left(X_{1} \times X_{2}, \tau_{1} \otimes \tau_{2}\right)$ is a compact metrizable space, we have $\mathcal{M}^{*}\left(X_{1} \times X_{2}\right)=\mathcal{M}\left(X_{1} \times X_{2}\right)$, and hence the implication follows from Proposition 2.2 and Theorem 3.13 .

ii) $\Rightarrow$ iii). Since $\left(X_{1}, \tau_{1}\right)$ and $\left(X_{2}, \tau_{2}\right)$ are assumed to be non-trivial, we find $\operatorname{dim} \mathcal{M}\left(X_{1}\right) \geq 2$ and $\operatorname{dim} \mathcal{M}\left(X_{2}\right) \geq 2$. Now iii) follows from Lemma 3.4 and Theorem 3.13.

iii) $\Rightarrow$ i). This can be shown by the theorem of Stone-Weierstraß, see e.g. Steinwart et al. (2016, Lemma A.5) for details.

Proof of Theorem 3.16: We write $E:=\operatorname{span}\left\{e_{i}: i \in I\right\}$ and denote the RKHS of $k$ by $H$.

ii) $\Leftrightarrow$ iii). Via the isomorphism (2.11) between $C_{0}(X)^{\prime}$ and $\mathcal{M}^{*}(X)$ we see that ii) is equivalent to the statement $\varphi_{\mid E}^{\prime}=0 \Longrightarrow \varphi^{\prime}=0$ for all $\varphi^{\prime} \in C_{0}(X)^{\prime}$ and by Hahn-Banach's theorem, see e.g. Dunford and Schwartz (1958, p. 64, Corollary II.3.13), the latter is equivalent to iii).

iii) $\Rightarrow$ i). The chain of inclusions $E \subset H \subset C_{0}(X)$ immediately gives the desired implication.

i) $\Rightarrow$ iii). Clearly, we have $E=\operatorname{span}\left\{\sqrt{\lambda_{i}} e_{i}: i \in I\right\}$ and since $\left(\sqrt{\lambda_{i}} e_{i}\right)_{i \in I}$ is an ONB of $H$, we conclude that $\bar{E}^{H}=H$. Let us now fix an $\varepsilon>0$ and an $f \in C_{0}(X)$. Since $k$ is universal, there then exists an $h \in H$ with $\|f-h\|_{\infty} \leq \varepsilon$ and for this $h$ our initial consideration yields an $e \in E$ with $\|e-h\|_{H} \leq \varepsilon$. Combining both estimates we find

$$
\|f-e\|_{\infty} \leq\|f-h\|_{\infty}+\|h-e\|_{\infty} \leq \varepsilon+\|k\|_{\infty}\|h-e\|_{H} \leq\left(1+\|k\|_{\infty}\right) \varepsilon,
$$

and hence $E$ is dense in $C_{0}(X)$.

To check the last statement, let us assume that $E$ is dense in $C_{0}(X)$. Using that $\nu$ is finite we then see that $E$ is also dense in $C_{0}(X)$ with respect to $\|\cdot\|_{L_{2}(\nu)}$, and since $C_{0}(X)$ is dense in $\mathcal{L}_{2}(\nu)$ by the regularity of $\nu$, see e.g. Bauer (2001, Theorem 29.14), we conclude that $E$ is dense in $\mathcal{L}_{2}(\nu)$. This shows that $[E]_{\sim}=\operatorname{span}\left\{\left[e_{i}\right]_{\sim}: i \in I\right\}$ is dense in $L_{2}(\nu)$, and therefore $\left(\left[e_{i}\right]_{\sim}\right)_{i \in I}$ is indeed an ONB of $L_{2}(\nu)$.

Proof of Corollary 3.17: i). Let us first assume that $\lambda_{i}>0$ for all $i \in I$. By Steinwart and Scovel (2012, Lemma 2.6 and Theorem 2.11) we then see that $k$ is of the form considered in Theorem 3.16, and since $\operatorname{span}\left\{e_{i}: i \in I\right\}$ is dense in $C(X)=C_{0}(X)$ by our assumption, we conclude that $k$ is universal. To show the converse implication we set $I^{*}:=\left\{i \in I: \lambda_{i}>0\right\}$ and assume that $k$ is universal but $I^{*} \neq I$. By the definition of $I^{*}$ we have

$$
k\left(x, x^{\prime}\right)=\sum_{i \in I^{*}} \lambda_{i} e_{i}(x) e_{i}\left(x^{\prime}\right)
$$

for all $x, x^{\prime} \in X$, and by Steinwart and Scovel (2012, Lemma 2.6 and Theorem 2.11) we thus conclude that $\left(e_{i}\right)_{i \in I *}$ is the family considered in Theorem 3.16. Consequently, this sub-family $\left(\left[e_{i}\right]_{\sim}\right)_{i \in I^{*}}$ is already an ONB in $L_{2}(\nu)$, which, however, is impossible for $I^{*} \neq I$.

ii). Let us first assume that $k$ is characteristic and that there exists an $j \neq i_{0}$ with $\lambda_{j}=0$. We have $\left[e_{j}\right]_{\sim} \in L_{1}(\nu)$ because $\nu(X)<\infty$. Moreover, $\left[e_{j}\right]_{\sim} \perp\left[e_{i}\right]_{\sim}$ for all $i \neq j$ implies both $\int_{X} e_{j} \mathrm{~d} \nu=0$ and $\left[e_{j}\right]_{\sim} \in[H]_{\sim}^{\perp}$, where in the last step we used (2.9). Consequently, $k$ cannot be characteristic by Theorem 3.9.

Conversely, assume that $\lambda_{i}>0$ for all $i \neq i_{0}$. If $\lambda_{i_{0}}>0$ then $k$ is actually universal by the already established part $i$ ), and thus characteristic. If $\lambda_{i_{0}}=0$, then the kernel

$$
k\left(x, x^{\prime}\right)+1=\sum_{i \neq i_{0}} \lambda_{i} e_{i}(x) e_{i}\left(x^{\prime}\right)+e_{i_{0}}(x) e_{i_{0}}\left(x^{\prime}\right)
$$


is universal by part $i$ ) and thus $k$ is characteristic by Theorem 3.13, Lemma 5.1, Proposition 2.2, and $\mathcal{M}^{*}(X)=\mathcal{M}(X)$.

iii). If $\lambda_{i}>0$ for all $i \in I$, then $i$ ) shows that $k$ is universal, and by Theorem 3.13, Proposition 2.2 , and $\mathcal{M}^{*}(X)=\mathcal{M}(X)$ we conclude that $k$ is characteristic. To show the converse implication, we assume that $k$ is characteristic and there is an $i_{0} \in I$ with $\lambda_{i_{0}}=0$. Since $\left(\left[e_{i}\right]_{\sim}\right)_{i \in I}$ is an ONS in $L_{2}(\nu)$ and $\left[e_{i_{0}}\right]_{\sim} \notin \overline{[H]_{\sim}}=\overline{\operatorname{span}\left\{\left[e_{i}\right]_{\sim}: \lambda_{i}>0\right\}}$, see $(2.9)$, we conclude that $\left[e_{i_{0}}\right]_{\sim} \in[H]_{\sim}^{\perp}$. On the other hand, $\mathbf{1}_{X} \in H$ gives $\left[\mathbf{1}_{X}\right]_{\sim} \in[H]_{\sim}$, and thus we find

$$
0=\left\langle\left[e_{i_{0}}\right]_{\sim},\left[\mathbf{1}_{X}\right]_{\sim}\right\rangle_{L_{2}(\nu)}=\int_{X} e_{i_{0}} \mathrm{~d} \nu .
$$

Finally, $\left[e_{i_{0}}\right]_{\sim} \neq 0$ is obvious and $\left[e_{i_{0}}\right]_{\sim} \in L_{1}(\nu)$ follows from $\nu(X)<\infty$, so that Theorem 3.9 shows that $k$ is not characteristic.

Proof of Corollary 3.18: Since compact Hausdorff spaces are normal, see Kelley (1955, p. 141), and thus completely regular, see Kelley (1955, p. 117), we know from Conway (1990, Theorem V.6.6.) that $C(X)$ is separable since $X$ is metrizable. Consequently, there exists a countable and dense subset $D \subset C(X)$ and without loss of generality we may additionally assume that $\mathbf{1}_{X} \in D$. By iteratively removing linearly dependent elements from $D$, we then find an at most countable family $\left(f_{i}\right)_{i \in I} \subset D$ with $\operatorname{span}\left\{f_{i}: i \in I\right\}=\operatorname{span} D$. Here, we follow our standard convention of at most countable index sets by assuming either $I=\mathbb{N}$ or $I=\{1, \ldots, n\}$. In addition, it is clear that by starting the iterative elimination process at $\mathbf{1}_{X} \in D$, we can guarantee that $f_{1}=\mathbf{1}_{X}$.

Our next goal is to construct a family $\left(e_{i}\right)_{i \in I}$ that satisfies the assumptions of Corollary 3.17. To this end, we fix an at most countable, dense family $\left(x_{j}\right)_{j \in J} \subset X$ of $X$ with $J=$ $\mathbb{N}$ or $J=\{1, \ldots, m\}$ Then $\nu:=\sum_{j \in J} 2^{-j} \delta_{x_{j}}$ is a strictly positive, finite, and regular Borel measure $X$. Now let $\left(\left[e_{i}\right]_{\sim}\right)_{i \in I}$ be the ONS in $L_{2}(\nu)$ that results from applying the Gram-Schmidt orthonormalization process to the family $\left(\left[f_{i}\right]_{\sim}\right)_{i \in I}$. Since $f_{i} \in C(X)$ for all $i \in I$, it is not hard to see that this process ensures that we can pick (actually unique) representatives $e_{i} \in C(X)$. In addition, $f_{1}=\mathbf{1}_{X}$ gives $e_{1}=\mathbf{1}_{X}$. It remains to show that $\operatorname{span}\left\{e_{i}: i \in I\right\}$ is dense in $C(X)$. However, this follows from

$$
\operatorname{span}\left\{e_{i}: i \in I\right\}=\operatorname{span}\left\{f_{i}: i \in I\right\}=\operatorname{span} D
$$

and the fact that $D$ and thus span $D$ is dense in $C(X)$.

Finally, to construct the desired kernel, we define $a_{i}:=\max \left\{\left\|e_{1}\right\|_{\infty}, \ldots,\left\|e_{i}\right\|_{\infty}\right\}$ for $i \in I$. Since all representatives $e_{i}$ are continuous, we find $a_{i}<\infty$ for all $i \in I$ and of course, the sequence $\left(a_{i}\right)_{i \in I}$ is increasing. We now define $\lambda_{1}:=0$ and $\lambda_{i}=2^{-i} a_{i}^{-2}$ for $i \in I \backslash\{1\}$. Clearly, $\left(\lambda_{i}\right)_{i \in I} \subset[0, \infty)$ is a family converging to 0 and a simple calculation gives

$$
\sum_{i \in I} \lambda_{i} e_{i}^{2}(x) \leq \sum_{i \in I \backslash\{1\}} 2^{-i} \frac{e_{i}^{2}(x)}{\left\|e_{i}\right\|_{\infty}^{2}}<\infty
$$

for all $x \in X$. By Steinwart and Scovel (2012, Lemma 2.6) we conclude that $k$ defined by (3.2) is a kernel on $X$. Moreover, if $I$ is finite, then $k$ is obviously continuous and if $I=\mathbb{N}$, then

$$
\left|k\left(x, x^{\prime}\right)-\sum_{i=2}^{n} \lambda_{i} e_{i}(x) e_{i}\left(x^{\prime}\right)\right|=\left|\sum_{i=n+1}^{\infty} \lambda_{i} e_{i}(x) e_{i}\left(x^{\prime}\right)\right| \leq \sum_{i=n+1}^{\infty} 2^{-i} \frac{e_{i}(x) e_{i}\left(x^{\prime}\right)}{\left\|e_{i}\right\|_{\infty}^{2}} \leq \sum_{i=n+1}^{\infty} 2^{-i},
$$

which holds for all $x, x^{\prime} \in X$, shows that the convergence in (3.2) is uniformly in $\left(x, x^{\prime}\right) \in X \times X$. Therefore, $k$ is continuous in this case, too. Now, we clearly have $i_{0}=1$, and therefore ii) of Corollary 3.17 guarantees that $k$ is characteristic. However, $k$ is not universal by $\lambda_{i_{0}}=0$ and $i$ ) of Corollary 3.17. 


\subsection{Proofs related to Section 4.1}

Proof of Lemma 4.1: To check that $\left(e_{i}^{*}\right)_{i \in I}$ is an ONS, we first observe that the equivalences $i=j \Leftrightarrow-i=-j$ and $i=-j \Leftrightarrow-i=j$ imply for $a_{i}, a_{j} \in\{-1,1\}$ that

$$
\begin{aligned}
& \left\langle\left[e_{i}+a_{i} \bar{e}_{i}\right]_{\sim},\left[e_{j}+a_{j} \bar{e}_{j}\right]_{\sim}\right\rangle_{L_{2}(G, \mathbb{C})} \\
& =\left\langle\left[e_{i}\right]_{\sim}+a_{i}\left[e_{-i}\right]_{\sim},\left[e_{j}\right]_{\sim}+a_{j}\left[e_{-j}\right]_{\sim}\right\rangle_{L_{2}(G, \mathbb{C})} \\
& =\left\langle\left[e_{i}\right]_{\sim},\left[e_{j}\right]_{\sim}\right\rangle+a_{j}\left\langle\left[e_{i}\right]_{\sim},\left[e_{-j}\right]_{\sim}\right\rangle+a_{i}\left\langle\left[e_{-i}\right]_{\sim},\left[e_{j}\right]_{\sim}\right\rangle+a_{i} a_{j}\left\langle\left[e_{-i}\right]_{\sim},\left[e_{-j}\right]_{\sim}\right\rangle \\
& =\left(1+a_{i} a_{j}\right) \delta_{i, j}+\left(a_{i}+a_{j}\right) \delta_{i,-j} .
\end{aligned}
$$

Let us first consider $i, j \in I_{0}$. Then we have $i=j$ if and only if $i=-j$, that is $\delta_{i, j}=\delta_{i,-j}$, and thus we find

$$
\left\langle\left[e_{i}^{*}\right]_{\sim},\left[e_{j}^{*}\right]_{\sim}\right\rangle_{L_{2}(G)}=\frac{1}{4}\left\langle\left[e_{i}+\bar{e}_{i}\right]_{\sim},\left[e_{j}+\bar{e}_{j}\right]_{\sim}\right\rangle_{L_{2}(G, \mathbb{C})}=\delta_{i, j}
$$

Similarly, for $i, j \in I_{+}$we cannot have $\delta_{i,-j}=1$, since this would imply $i \in I_{-}$, and hence we obtain

$$
\left\langle\left[e_{i}^{*}\right]_{\sim},\left[e_{j}^{*}\right]_{\sim}\right\rangle_{L_{2}(G)}=\frac{1}{2}\left\langle\left[e_{i}+\bar{e}_{i}\right]_{\sim},\left[e_{j}+\bar{e}_{j}\right]_{\sim}\right\rangle_{L_{2}(G, \mathbb{C})}=\delta_{i, j},
$$

and for $i, j \in I_{-}$we find by an analogous reasoning that

$$
\left\langle\left[e_{i}^{*}\right]_{\sim},\left[e_{j}^{*}\right]_{\sim}\right\rangle_{L_{2}(G)}=\frac{1}{2}\left\langle\left[e_{i}-\bar{e}_{i}\right]_{\sim},\left[e_{j}-\bar{e}_{j}\right]_{\sim}\right\rangle_{L_{2}(G, \mathbb{C})}=\delta_{i, j} .
$$

For the mixed cases, in which $i$ and $j$ belong to different partition elements $I_{0}, I_{+}$, or $I_{-}$we clearly have $i \neq j$, and hence the above calculation reduces to

$$
\left\langle\left[e_{i}+a_{i} \bar{e}_{i}\right]_{\sim},\left[e_{j}+a_{j} \bar{e}_{j}\right]_{\sim}\right\rangle_{L_{2}(G, \mathbb{C})}=\left(a_{i}+a_{j}\right) \delta_{i,-j} .
$$

Now, if $i \in I_{0}$ and $j \notin I_{0}$, then we cannot have $i=-j$ and hence we obtain $\left\langle\left[e_{i}^{*}\right]_{\sim},\left[e_{j}^{*}\right]_{\sim}\right\rangle_{L_{2}(G)}=0$. For the same reason we find for $i \in I_{+}$and $j \in I_{-}$with $i \neq-j$ that $\left\langle\left[e_{i}^{*}\right]_{\sim},\left[e_{j}^{*}\right]_{\sim}\right\rangle_{L_{2}(G)}=0$. Finally, for $i \in I_{+}$and $j \in I_{-}$with $i=-j$ we have

$$
\left\langle\left[e_{i}^{*}\right]_{\sim},\left[e_{j}^{*}\right]_{\sim}\right\rangle_{L_{2}(G)}=\frac{1}{2}\left\langle\left[e_{i}+\bar{e}_{i}\right]_{\sim}, \mathrm{i} \cdot\left[e_{j}-\bar{e}_{j}\right]_{\sim}\right\rangle_{L_{2}(G, \mathbb{C})}=-\mathrm{i} \cdot(1-1) \cdot \delta_{i,-j}=0,
$$

and therefore we conclude that $\left(\left[e_{i}^{*}\right]_{\sim}\right)_{i \in I}$ is an ONS in $L_{2}(G)$.

Our next goal is to show that $\operatorname{span}\left\{e_{i}^{*}: i \in I\right\}$ is dense in $C(G)$. To this end, we fix an $f \in C(G)$ and an $\varepsilon>0$. Since $f \in C(G, \mathbb{C})$ and $\left(e_{i}\right)_{i \in I}$, is dense in $C(G, \mathbb{C})$, there then exists a finite set $J \subset I$ and $\left(a_{j}\right)_{j \in J} \subset \mathbb{C}$ such that

$$
\sup _{x \in G}\left|\sum_{j \in J} a_{j} e_{j}(x)-f(x)\right|<\varepsilon,
$$

and from this conclude that

$$
\begin{aligned}
\sup _{x \in G}\left|\sum_{j \in J} \operatorname{Re} a_{j} \operatorname{Re} e_{j}(x)-\sum_{j \in J} \operatorname{Im} a_{j} \operatorname{Im} e_{j}(x)-f(x)\right| & =\sup _{x \in G}\left|\operatorname{Re}\left(\sum_{j \in J} a_{j} e_{j}(x)\right)-\operatorname{Re} f(x)\right| \\
& \leq \sup _{x \in G}\left|\sum_{j \in J} a_{j} e_{j}(x)-f(x)\right| \\
& <\varepsilon .
\end{aligned}
$$


In other words, the span of $\left(\operatorname{Re} e_{i}\right)_{i \in I} \cup\left(\operatorname{Im} e_{i}\right)_{i \in I}$ is dense in $C(G)$. However, our initial considerations showed that $\operatorname{Re} e_{i}=\operatorname{Re} e_{-i}$ and $\operatorname{Im} e_{i}=-\operatorname{Im} e_{-i}$ for all $i \in I$ as well as $\operatorname{Im} e_{i}=0$ for all $i \in I_{0}$, and therefore $\operatorname{span}\left\{e_{i}^{*}: i \in I\right\}$ is dense in $C(G)$, too.

Finally, $\nu$ is regular, and therefore $C(G)$ is dense in $L_{2}(G)$, see e.g. Bauer (2001, Theorem 29.14). Consequently, $\operatorname{span}\left\{e_{i}^{*}: i \in I\right\}$ is dense in $L_{2}(G)$, and therefore $\left(e_{i}^{*}\right)_{i \in I}$ is an ONB of $L_{2}(G)$. The estimate $\left\|e_{i}^{*}\right\|_{\infty} \leq \sqrt{2}$ follows from $\left|e_{i}(x)\right|=1$ for all $x \in G$.

Proof of Lemma 4.2: Let $\nu$ be the Haar measure of $G$.

i) $\Rightarrow$ ii). For a character $e_{i} \in \hat{G}$ and $x \in G$ a simple calculation shows

$$
\int_{G} k\left(x, x^{\prime}\right) e_{i}\left(x^{\prime}\right) \mathrm{d} \nu\left(x^{\prime}\right)=\int_{G} \kappa\left(-x+x^{\prime}\right) e_{i}\left(x^{\prime}\right) \mathrm{d} \nu\left(x^{\prime}\right)=\int_{G} \kappa\left(x^{\prime}\right) e_{i}\left(x+x^{\prime}\right) \mathrm{d} \nu\left(x^{\prime}\right)=\lambda_{i} e_{i}(x),
$$

where $\lambda_{i}:=\int_{G} \kappa\left(x^{\prime}\right) e_{i}\left(x^{\prime}\right) \mathrm{d} \nu\left(x^{\prime}\right)$. Now, since $k$ is $\mathbb{R}$-valued, the integral operator $T_{k}^{\mathbb{C}}: L_{2}(G, \mathbb{C}) \rightarrow$ $L_{2}(G, \mathbb{C})$ is self-adjoint and the previous calculation shows that each $e_{i}$ gives an eigenvector $\left[e_{i}\right]_{\sim} \in L_{2}(G, \mathbb{C})$ of $T_{k}^{\mathbb{C}}$ with eigenvalue $\lambda_{i}$. Using that eigenvalues of self-adjoint operators are real numbers, we then find

$$
\begin{aligned}
T_{k}\left[\operatorname{Re} e_{i}\right]_{\sim} & =T_{k}^{\mathbb{C}}\left[\operatorname{Re} e_{i}\right]_{\sim}=\lambda_{i}\left[\operatorname{Re} e_{i}\right]_{\sim} \\
T_{k}\left[\operatorname{Im} e_{i}\right]_{\sim} & =T_{k}^{\mathbb{C}}\left[\operatorname{Im} e_{i}\right]_{\sim}=\lambda_{i}\left[\operatorname{Im} e_{i}\right]_{\sim}
\end{aligned}
$$

for all $i \in I$. Now recall that for $i \in I_{0}$ we have $\operatorname{Im} e_{i}=0$ and therefore these functions $\operatorname{Im} e_{i}$ are not eigenvectors of $T_{k}: L_{2}(G) \rightarrow L_{2}(G)$. By Lemma 4.1 we thus conclude that $\left(\left[e_{i}^{*}\right]_{\sim}\right)_{i \in I}$ is an ONB of eigenvectors of $T_{k}$ with corresponding eigenvalues $\left(\lambda_{i}\right)_{i \in I}$, and in particular, there are no further eigenvalues than these. Moreover, $\left(\lambda_{i}\right)_{i \in I}$ is summable since (2.8) holds. Moreover, for $i \in I$ we have $\lambda_{i}=\lambda_{-i}$, where we note that for $i \notin I_{0}$ the corresponding eigenvalues have thus a geometric multiplicity of at least two. Since $\nu$ is finite and strictly positive, we thus see that $k$ enjoys a Mercer representation (2.10) for the index set $I^{*}:=\left\{i \in I: \lambda_{i}>0\right\}$ and the sub-family $\left(e_{i}^{*}\right)_{i \in I^{*}}$. Moreover, for $x, x^{\prime} \in G$ this representation yields

$$
\begin{aligned}
k\left(x, x^{\prime}\right)=\sum_{\lambda_{i}>0} \lambda_{i} e_{i}^{*}(x) e_{i}^{*}\left(x^{\prime}\right)= & \sum_{i \in I_{0}: \lambda_{i}>0} \lambda_{i} \operatorname{Re} e_{i}(x) \operatorname{Re} e_{i}\left(x^{\prime}\right) \\
& +2 \sum_{i \in I_{+}: \lambda_{i}>0} \lambda_{i}\left(\operatorname{Re} e_{i}(x) \operatorname{Re} e_{i}\left(x^{\prime}\right)+\operatorname{Im} e_{i}(x) \operatorname{Im} e_{i}\left(x^{\prime}\right)\right) \\
= & \sum_{i \in I_{0}: \lambda_{i}>0} \lambda_{i} \operatorname{Re} e_{i}\left(-x^{\prime}+x\right)+2 \sum_{i \in I_{+}: \lambda_{i}>0} \lambda_{i} \operatorname{Re} e_{i}\left(-x^{\prime}+x\right) \\
= & \sum_{\lambda_{i}>0} \lambda_{i} \operatorname{Re} e_{i}\left(-x^{\prime}+x\right),
\end{aligned}
$$

where in the second to last step we used (4.1) and the last step rests on $\operatorname{Re} e_{i}=\operatorname{Re} e_{-i}$. In addition, $\sup _{i \in I}\left\|e_{i}^{*}\right\|_{\infty} \leq \sqrt{2}$ together with the summability of $\left(\lambda_{i}\right)_{i \in I}$ quickly shows that the series converge both absolutely and uniformly. Finally, the continuity of $k$ follows from the uniform convergence in (4.3) and $e_{i}^{*} \in C(G)$ for all $i \in I$.

ii) $\Rightarrow$ i). We first observe that Lemma 4.1 together with Steinwart and Scovel (2012, Lemma 2.6) shows that (4.3) does indeed define a kernel $k$, and its translation invariance is built into the construction. Clearly, $k$ is measurable and $\sup _{i \in I}\left\|e_{i}^{*}\right\|_{\infty} \leq \sqrt{2}$ together with the summability of $\left(\lambda_{i}\right)_{i \in I}$ shows that $k$ is bounded.

Proof of Theorem 4.3: The equivalences i) $\Leftrightarrow i v) \Leftrightarrow v i$ ) have already been shown in Theorem 3.14 , and $i i i) \Rightarrow i v$ ) is trivial. In addition, if one of the conditions iii) - vi) are satisfied, Theorem 
3.14 shows that $\mathcal{M}^{*}(G)=\mathcal{M}(G)$, where in the case of $v$ ) we additionally need Lemma 4.2. Therefore Lemma 5.1 together with Proposition 2.2 and Theorem 3.13 shows that a continuous kernel $k$ is characteristic if and only if $k+1$ is universal. This yields $i v) \Leftrightarrow v i$ ), and by the last statement in Lemma 4.2 , also iii) $\Leftrightarrow v$ ). It thus suffices to show ii) $\Rightarrow$ iii) and $i) \Rightarrow$ ii).

ii) $\Rightarrow$ iii). If $\hat{G}$ is at most countable, so is $I$, and hence there exists a family $\left(\lambda_{i}\right)_{i \in I}$ with $\lambda_{i}>0$ for all $i \in I$ and $\sum_{i \in I} \lambda_{i}<\infty$. The kernel $k$, which is constructed by (4.3), is then translation-invariant and continuous, and by Corollary 3.17 it is also universal.

i) $\Rightarrow$ ii). By Schurle (1979, Theorem 3.2.11 and Corollary 3.3.2) we know that $G$ is completely regular and hence Conway (1990, Theorem V.6.6.) shows that $G$ is metrizable if and only if $C(G)$ is separable. We therefore see that $C(G)$ is separable. In addition, since $\nu$ is regular, Bauer $(2001$, Theorem 29.14) shows that $C(G)$ is dense in $L_{2}(G)$. Consequently, $L_{2}(G)$ is separable, and since $\left(e_{i}^{*}\right)_{i \in I}$ is an ONB of $L_{2}(G)$, we conclude that $I$, and thus $\hat{G}$, is at most countable.

Proof of Corollary 4.4: Since the Haar measure $\nu$ on $G$ is a finite, regular, and strictly positive Borel measure, we see by Lemma 4.1 that all assumptions of Corollary 3.17 are satisfied. Moreover, we have $e_{0}^{*}=\mathbf{1}_{G}$. Now the assertions follow from Corollary 3.17.

\subsection{Proofs related to Section 4.2}

Proof of Proof of Theorem 4.5: We first consider the case $\psi \in \Psi_{d+2}$. If the kernel $k$ on $\mathbb{S}^{d}$ induced by $\psi$ is strictly positive definite then Lemma 4.9 implies that $b_{n, d}>0$ for all $n \geq 0$. By Theorem 4.11, $k$ is then universal and characteristic. Conversely, if $k$ is universal or characteristic then $b_{n, d}>0$ for all $n \geq 1$ by Theorem 4.11, thus it is strictly positive definite by Remark 4.8.

In the case $\psi \in \Psi_{d+1}^{+}$, we have $\psi \in \Psi_{d}^{+}$, and hence it suffices to show that $k$ is universal and characteristic. This, however, follows from Lemma 4.10 and Theorem 4.11.

Proof of Theorem 4.6: Suppose that the kernel $k$ is induced by $\psi \in \Psi_{\infty}^{+}$. Then $b_{n, d}>0$ for all $n \in \mathbb{N}_{0}$ by Lemma 4.10 and hence the kernel is universal and characteristic by Theorem 4.11. Suppose now that $k$ is characteristic. By Proposition 4.13 we obtain that $\psi \in \Psi_{\infty}^{+}$.

Proof of Lemma 4.9: It is clear from the results of (Chen et al., 2003) that $b_{n, d}>0$ for all $n \in \mathbb{N}_{0}$ is a sufficient condition for $\psi$ being strictly positive definite. Suppose now that $\psi \in \Psi_{d+2} \cap \Psi_{d}^{+}$. Gneiting (2013, Corollary 4) implies that if $b_{2 k+2, d}>0\left(b_{2 k+1, d}>0\right)$ for some $k$, then $b_{2 k^{\prime}+2, d}>0$ $\left(b_{2 k^{\prime}+1, d}>0\right)$ for all $k^{\prime} \leq k$. This yields the claim by Remark 4.8.

Proof of Lemma 4.10: This is shown in Gneiting (2013, Proof of Corollary 1(b)).

Proof of Corollary 4.12: This follows from Theorem 4.11 and Remark 4.8.

Proof of Proposition 4.13: Assume that $\psi$ is not strictly positive definite, or, by Remark 4.8, does not satisfy condition $b$. We will show that it cannot be characteristic.

First, we construct a special class of probability densities $p$ on $\mathbb{S}^{d}$ such that we explicitly know the integrals

$$
c_{k, j}(p):=\int_{\mathbb{S}^{d}} e_{k, j}(y) p(y) \mathrm{d} \sigma(y)
$$

with respect to the basis of spherical harmonics. Here, $\sigma$ is the surface area measure on $\mathbb{S}^{d}$ normalized such that $\int_{\mathbb{S}^{d}} \mathrm{~d} \sigma=1$. Fix $v_{0} \in \mathbb{S}^{d}$ and $a \in[-1,1] \backslash\{0\}$. We have

$$
\left|C_{n}^{(d-1) / 2}(x)\right| \leq C_{n}^{(d-1) / 2}(1), \quad x \in[-1,1],
$$


see (DLMF, 18.14.4 for $d \geq 2$ ), and therefore, for all $n \geq 1, x \in \mathbb{S}^{d}$, we have

$$
p_{n, a}(x):=1+a \frac{C_{n}^{(d-1) / 2}\left(\left\langle v_{0}, x\right\rangle\right)}{C_{n}^{(d-1) / 2}(1)} \geq 0,
$$

and $\int_{\mathbb{S}^{d}} p_{n, a}(x) \mathrm{d} \sigma(x)=1$, where for the last equality we used that $C_{n}^{(d-1) / 2}\left(\left\langle v_{0}, \cdot\right\rangle\right)$ is a spherical harmonics of degree $n$ and thus orthogonal to $e_{0,0}=\mathbf{1}_{\mathbb{S}^{d}}$. Consequently, $p_{n, a}$ is a probability density function on $\mathbb{S}^{d}$ with respect to the surface area measure $\sigma$. Note that $p_{n, a}$ and $p_{n^{\prime}, a}$ induce different probability measures on $\mathbb{S}^{d}$ for $n \neq n^{\prime}$. We obtain

$$
c_{k, j}\left(p_{n, a}\right)=\delta_{k, 0}+\delta_{k, n} \frac{a}{N(d, k)} e_{k, j}\left(v_{0}\right)
$$

using (4.6), and the Funk-Hecke Theorem (Groemer, 1996, Theorem 3.4.1) yields that

$$
\int_{\mathbb{S}^{d}}\langle x, y\rangle^{n} e_{k, j}(y) \mathrm{d} \sigma(y)=\lambda_{k}^{n} e_{k, j}(x), \quad x \in \mathbb{S}^{d}, j=1, \ldots, N(d, k),
$$

where

$$
\lambda_{k}^{n}=\frac{\Gamma((d+1) / 2)}{\sqrt{\pi} \Gamma(d / 2)} C_{k}^{(d-1) / 2}(1)^{-1} \int_{-1}^{1} t^{n} C_{k}^{(d-1) / 2}(t)\left(1-t^{2}\right)^{(d-2) / 2} d t .
$$

Since the family $\left(e_{n, j}\right)_{n \in \mathbb{N}_{o}, j=1, \ldots, N(d, k)}$ is an ONB of $L_{2}\left(\mathbb{S}^{d}\right)$, we obtain the following Mercer representation of the bounded and continuous kernel $(x, y) \mapsto\langle x, y\rangle^{n}$ on $\mathbb{S}^{d}$

$$
\langle x, y\rangle^{n}=\sum_{k=0}^{\infty} \sum_{j=1}^{N(d, k)} \lambda_{k}^{n} e_{k, j}(x) e_{k, j}(y)
$$

where for each $x$ the convergence is uniform in $y$ by Steinwart and Scovel (2012, Corollary 3.5). Using that $C_{k}^{(d-1) / 2}$ is even for $k$ even and odd for $k$ odd, one obtains that $\lambda_{k}^{n}=0$ if $k-n$ is odd. The $C_{k}^{(d-1) / 2}$ are orthogonal with respect to the weight function $\left(1-t^{2}\right)^{(d-2) / 2}$ DLMF, 18.3.1, therefore $\lambda_{k}^{n}=0$ for $k>n$. Finally, the formula DLMF, 18.17.37 for the Mellin transform yields that

$$
\lambda_{k}^{n}=\frac{\pi 2^{d-n-1} \Gamma(k+d-1) \Gamma(n+1)}{k ! \Gamma\left(\frac{d-1}{2}\right) \Gamma\left(\frac{k+d+n}{2}\right) \Gamma\left(\frac{n-k+2}{2}\right)}>0 \quad k \leq n, k-n \text { even. }
$$

For a probability density $p$ on $\mathbb{S}^{d}$, we have by (4.5) and (5.4) for $x \in \mathbb{S}^{d}$,

$$
\int_{\mathbb{S}^{d}} k(x, y) p(y) \mathrm{d} \sigma(y)=\sum_{n=0}^{\infty} \sum_{k=0}^{n} \sum_{j=1}^{N(d, k)} b_{n} \lambda_{k}^{n} e_{k, j}(x) c_{k, j}(p)=\sum_{k=0}^{\infty} z_{k} \sum_{j=1}^{N(d, k)} c_{k, j}(p) e_{k, j}(x),
$$

where $z_{k}=\sum_{n=k}^{\infty} b_{n} \lambda_{k}^{n}$ and $c_{k, j}(p)$ is defined at (5.1). If $b_{n}=0$ for all even $n \geq n_{0}$ then $z_{k}=0$ for all even $k \geq n_{0}$. If $b_{n}=0$ for all odd $n \geq n_{0}$ then $z_{k}=0$ for all odd $k \geq n_{0}$.

Let us start with the case that $b_{n}=0$ for all even $n \geq n_{0}$, i.e. $z_{k}=0$ for all even $k \geq n_{0}$. For all $m \in \mathbb{N}_{0}$, we have $c_{k, j}\left(p_{2 m, a}\right)=0$ for $k$ odd by (5.3), where $p_{n, a}$ is defined at (5.2). Hence, for $2 m \geq n_{0}$ and $x \in \mathbb{S}^{d}$, we obtain

$$
\int_{\mathbb{S}^{d}} k(x, y) p_{2 m, a}(y) \mathrm{d} \sigma(y)=\sum_{k=0, k \text { even }}^{n_{0}} z_{k} \sum_{j=1}^{N(d, k)}\left(\delta_{k, 0}+\delta_{k, 2 m} \frac{a}{N(d, k)} e_{k, j}\left(v_{0}\right)\right) e_{k, j}(x)=z_{0},
$$


which shows that the kernel mean embedding maps all these densities to the constant function with value $z_{0}$. Consequently, $k$ is not characteristic.

Suppose now that $b_{n}=0$ for all odd $n \geq n_{0}$, i.e. $z_{k}=0$ for all odd $k \geq n_{0}$. For all $m \in \mathbb{N}_{0}$, we have $c_{k, j}\left(p_{2 m+1, a}\right)=\delta_{k, 0}$ for $k$ even by (5.3). Hence, for $2 m+1 \geq n_{0}$ and $x \in \mathbb{S}^{d}$, we obtain

$$
\int_{\mathbb{S}^{d}} k(x, y) p_{2 m+1, a}(y) \mathrm{d} \sigma(y)=z_{0}+\sum_{k=1, k \text { odd }}^{n_{0}} z_{k} \sum_{j=1}^{N(d, k)} \delta_{k, 2 m+1} \frac{a}{N(d, k)} e_{k, j}\left(v_{0}\right) e_{k, j}(x)=z_{0},
$$

which again shows that the kernel mean embedding maps all these densities to the constant function with value $z_{0}$. Consequently, $k$ is not characteristic.

\section{References}

H. Bauer. Measure and Integration Theory. De Gruyter, Berlin, 2001.

C. Berg, J. P. R. Christensen, and P. Ressel. Harmonic Analysis on Semigroups. Springer, New York, 1984.

S. Bochner. Hilbert distances and positive definite functions. Ann. of Math., 42:647-656, 1941.

D. Chen, V. A. Menegatto, and X. Sun. A necessary and sufficient condition for strictly positive definite functions on spheres. Proc. Amer. Mat. Soc., 131:2733-2740, 2003.

W. Chen, B. Wang, and H. Zhang. Universalities of reproducing kernels revisited. Appl. Anal., 95:1776$1791,2016$.

J. B. Conway. A Course in Functional Analysis. Springer, New York, 2nd edition, 1990.

J. Diestel and J. J. Uhl. Vector Measures. American Mathematical Society, Providence, 1977.

J. Diestel, H. Jarchow, and A. Tonge. Absolutely Summing Operators. Cambridge University Press, Cambridge, 1995.

Digital Library of Mathematical Functions (DLMF). Release date 2011-08-29. National Institute of Standards and Technology from http://dlmf.nist.gov/, 2011.

N. Dunford and J. T. Schwartz. Linear Operators, Part I: General Theory. Interscience Publishers, New York, 1958

G. B. Folland. A course in Abstract Harmonic Analysis. CRC Press, Boca Raton, 1995.

B. Fuglede. On the theory of potentials in locally compact spaces. Acta Math., 103:139-215, 1960.

K. Fukumizu, A. Gretton, X. Sun, and B. Schölkopf. Kernel measures of conditional dependence. In J. C. Platt, D. Koller, Y. Singer, and S. T. Roweis, editors, Advances in Neural Information Processing Systems 20, pages 489-496, 2008.

K. Fukumizu, B. Sriperumbudur, A. Gretton, and B. Schölkopf. Characteristic kernels on groups and semigroups. In D. Koller, D. Schuurmans, Y. Bengio, and L. Bottou, editors, Advances in Neural Information Processing Systems 21, pages 473-480, 2009.

T. Gneiting. Strictly and non-strictly positive definite functions on spheres. Bernoulli, 19:1327-1349, 2013.

T. Gneiting and M. Katzfuss. Probabilistic forecasting. Ann. Rev. Stat. Appl., 1:125-151, 2014. 
T. Gneiting and A. E. Raftery. Strictly proper scoring rules, prediction, and estimation. J. Amer. Statist. Assoc., 102:359-378, 2007.

A. Gretton, K.M. Borgwardt, M. Rasch, B. Schölkopf, and A.J. Smola. A kernel method for the two-sampleproblem. In P. B. Schölkopf, J. C. Platt, and T. Hoffman, editors, Advances in Neural Information Processing Systems 19, pages 513-520, 2007.

H. Groemer. Geometric applications of Fourier series and sperical harmonics. Cambridge University Press, New York, 1996.

E. Hewitt and K.A. Ross. Abstract Harmonic Analysis. Vol. I. Springer, Berlin, 1963.

E. Hewitt and K.A. Ross. Abstract Harmonic Analysis. Vol. II. Springer, Berlin, 1970.

E. Hewitt and K. Stromberg. Real and Abstract Analysis. A Modern Treatment of the Theory of Functions of a Real Variable. Springer, New York, 1965.

K.H. Hofmann and S.A. Morris. The Structure of Compact Groups: A Primer for the Student - A Handbook for the Expert. De Gruyter, Berlin, 3rd edition, 2013.

J. L. Kelley. General Topology. D. Van Nostrand, Toronto, 1955.

N. S. Landkof. Foundations of Modern Potential Theory. Springer, New York, 1972.

R. E. Megginson. An Introduction to Banach Space Theory. Springer, New York, 1998.

V. A. Menegatto. Interpolation on spherical spaces. PhD thesis, University of Texas at Austin, 1992.

V. A. Menegatto. Strictly positive definite kernels on the Hilbert sphere. Appl. Anal., 55:91-101, 1994.

V. A. Menegatto. Strictly positive definite kernels on the circle. Rocky Mountain J. Math., 25:1149-1163, 1995.

V. A. Menegatto, C. P. Oliveira, and A. P. Peron. Strictly positive definite kernels on subsets of the complex plane. Comput. Math. Appl., 51:1233-1250, 2006.

C. A. Micchelli, Y. Xu, and H. Zhang. Universal kernels. J. Mach. Learn. Res., 7:2651-2667, 2006.

S.A. Morris. Duality and structure of locally compact Abelian groups...for the layman. Mathematical Chronicle, 8:39-56, 1979.

K. Muandet, K. Fukumizu, B. Sriperumbudur, and B. Schölkopf. Kernel mean embedding of distributions: A review and beyond. Foundations and Trends in Machine Learning, 10:1-141, 2017.

N.S. Pillai, Q. Wu, F. Liang, S. Mukherjee, and R.L. Wolpert. Characterizing the function space for Bayesian kernel models. J. Mach. Learn. Res., 8:1769-1797, 2007.

I. J. Schoenberg. Positive definite functions on spheres. Duke Math. J., 9:96-108, 1942.

A.W. Schurle. Topics in Topology. Elsevier North Holland, 1979.

C.-J. Simon-Gabriel and B. Schölkopf. Kernel distribution embeddings: Universal kernels, characteristic kernels and kernel metrics on distributions. J. Mach. Learn. Res., 19(44):1-29, 2018.

B. K. Sriperumbudur, A. Gretton, K. Fukumizu, B. Schölkopf, and G. R. G. Lanckriet. Hilbert space embeddings and metrics on probability measures. J. Mach. Learn. Res., 11:1517-1561, 2010a.

B. K. Sriperumbudur, K. Fukumizu, and G. R. G. Lanckriet. Universality, characteristic kernels and RKHS embedding of measures. J. Mach. Learn. Res., 12:2389-2410, 2011. 
B.K. Sriperumbudur, K. Fukumizu, and G. Lanckriet. On the relation between universality, characteristic kernels and RKHS embedding of measures. In Y.W. Teh and D.M. Titterington, editors, Proceedings of the Thirteenth International Conference on Artificial Intelligence and Statistics, volume 9 of Proceedings of Machine Learning Research, pages 773-780, 2010b.

I. Steinwart. On the influence of the kernel on the consistency of support vector machines. J. Mach. Learn. Res., 2:67-93, 2001.

I. Steinwart and A. Christmann. Support Vector Machines. Springer, New York, 2008.

I. Steinwart and C. Scovel. Mercer's theorem on general domains: on the interaction between measures, kernels, and RKHSs. Constr. Approx., 35:363-417, 2012.

I. Steinwart, D. Hush, and C. Scovel. Function classes that approximate the Bayes risk. In G. Lugosi and H. U. Simon, editors, Proceedings of the 19th Annual Conference on Learning Theory, pages 79-93, 2006.

I. Steinwart, P. Thomann, and N. Schmid. Learning with hierarchical Gaussian kernels. Technical report, Fakultät für Mathematik und Physik, Universität Stuttgart, 2016. URL http://arxiv.org/abs/1612.00824.

X. Sun and E. W. Cheney. Fundamental sets of continuous functions on spheres. Constr. Approx., 13: 245-250, 1997. 